\title{
The Earth Mover's Distance as a Metric for the Space of Inorganic Compositions
}

Cameron J. Hargreaves, ${ }^{1,2}{ }^{*}$ Matthew S. Dyer, ${ }^{1}$ Michael W. Gaultois,, ${ }^{1}$ Vitaliy A. Kurlin,, 3 Matthew J. Rosseinsky ${ }^{1,3}$

${ }^{1}$ Department of Chemistry, University of Liverpool, Liverpool L69 7ZD, United Kingdom, ${ }^{2}$ Department of Computer Science, University of Liverpool, ${ }^{3}$ Leverhulme Research Center for Functional Material Design, Materials Innovation Factory, University of Liverpool

\section{Supplementary Information}




\section{Commentary on the non-Euclidean structure of inorganic compositional space with respect to the EMD.}

From an early point of geometric education, we are taught that the sum of interior angles for a triangle is $180^{\circ}$, and in most everyday use this statement holds. It is not difficult however to construct a real world experiment which directly violates this condition. Starting from the equator, one can travel north continuously for approximately 10,000 km, before making an exact right hand turn when you have reached the north pole. Travelling for a further 10,000 km, you would find yourself approaching the equator not only at an exact right angle, but also $10,000 \mathrm{~km}$ east from your starting position. We may observe that these two points on the equator and the pole form a triangle, where the sum of interior angles for our path has come to $270^{\circ}$. This well-known phenomena is due to the positive curvature of the Earth's surface, and from manifold theory we find that as we either increase these edge lengths, or apply more curvature, the sum of interior angles further increases (although the resultant global structure will no longer be perfectly spherical).

It is presumptuous to assume that an arbitrary metric space will have an associated geometry, which is to say, angles between edges which meet at a point are not guaranteed to exist. In graph theory for example, a defining feature is the lack of angle between edges, yet analyzing the topological structure of these networks remains a powerful tool. For a set of objects with an associated metric, any two objects will have a distance of fixed length, and we may call this metric space with its topological structure a length space. Given three points, there is human tendency to associate with these a geometric triangle, but we do not know that a length space carries the formal prerequisites to make such a connection. Formally defining such notions has motivated the field of Alexandrov geometry, where the concept of curvature for general metric spaces was first introduced in 1951 [1], and further advances have generalized this to the theory of CAT $(\mathrm{k})$ spaces.

The Earth Mover's Distance as it is normally referred to in computer science has been rediscovered many times, giving it a rich, but scattered history. In mathematics it is most commonly called the Wasserstein distance (specifically in this instance the 1Wasserstein distance), however other names include the Gini distance, the Monge-Kantorovich problem, the Monge-Rubinstein metric, and the Mallows distance. The Earth Movers Distance is additionally used in statistics to determine the similarity of probability density functions. Given its wide applicability it is unsurprising that the structure has been well studied, and this metric space has been determined to always possess positive curvature [2]. A complete overview of the subject including the methods to analytically derive this curvature is given in Cédric Villanis treatise "Optimal Transport: Old and New" [3]. 


\section{Structure Maps under Alternate Compositional Vector Representations}
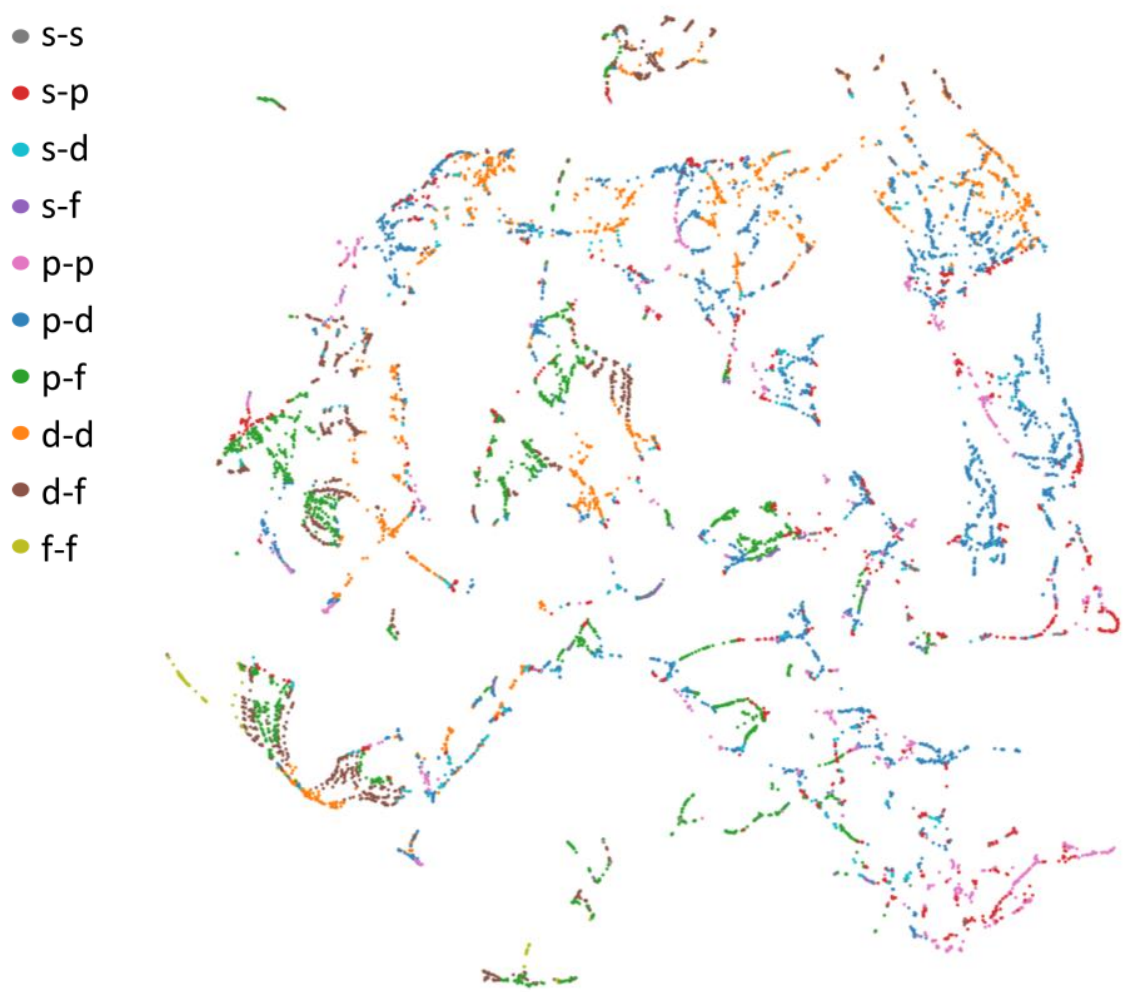

Figure S1. The Atomic Scale - Given the standard numbering of elements as our index for composition vectors, we see that there is structure in the embedding, however each of the clusters has much greater impurity in chemical labels
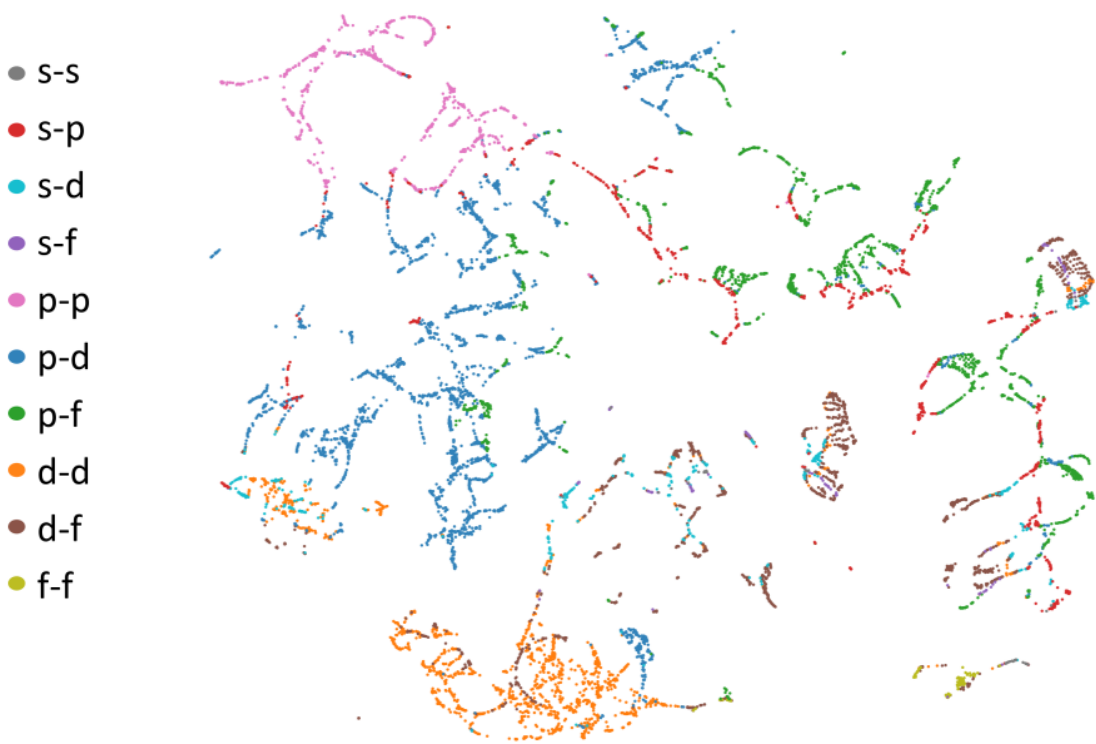
Figure S2. Pettifor's Mendeleev Number [4] - Using the number scale originally devised by Pettifor we see that we regain the clear separation into major clusters of chemical labels.

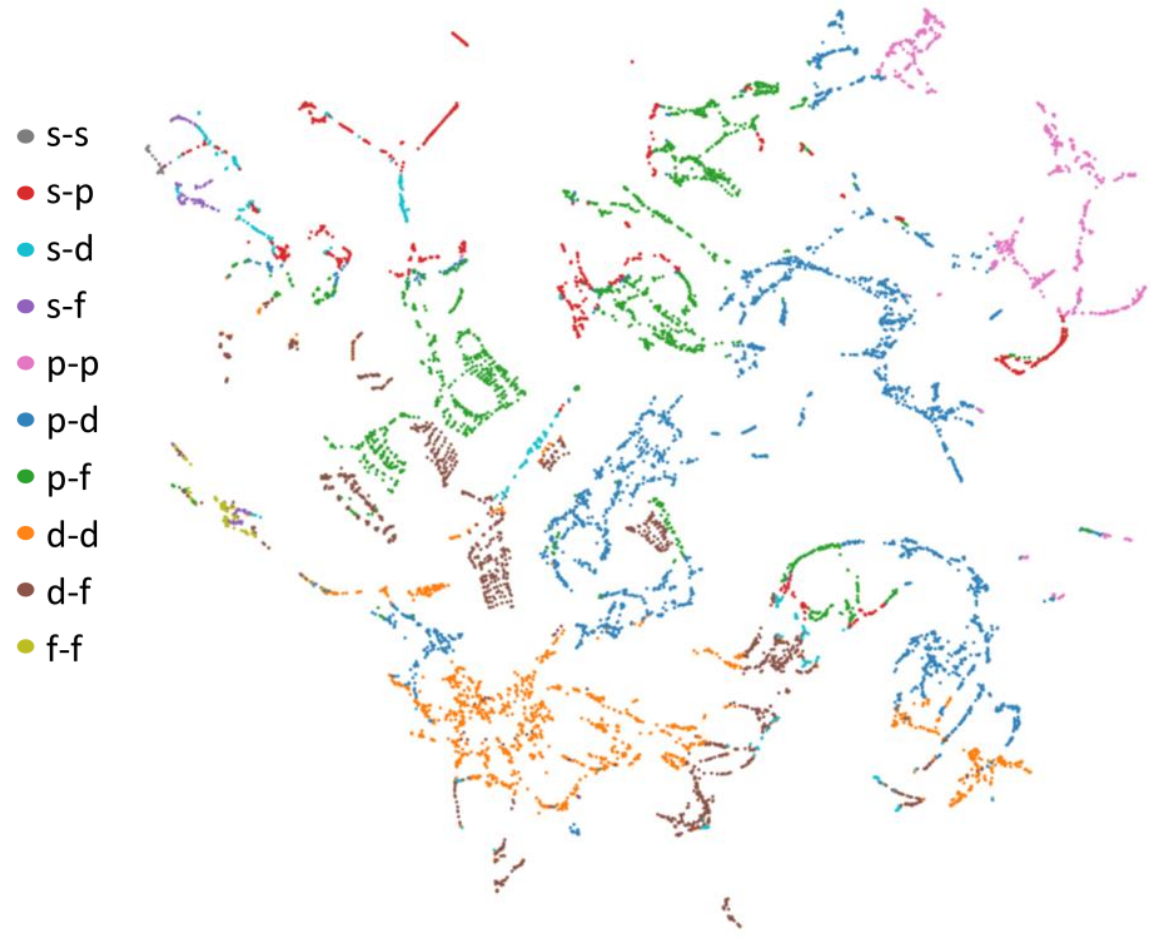

Figure S3. The Mendeleev Number (Villars first ordering)[5] - This ordering follows the periodic table from left to right, top to bottom, and preserves much of the same structure as Pettifor's initial scale.
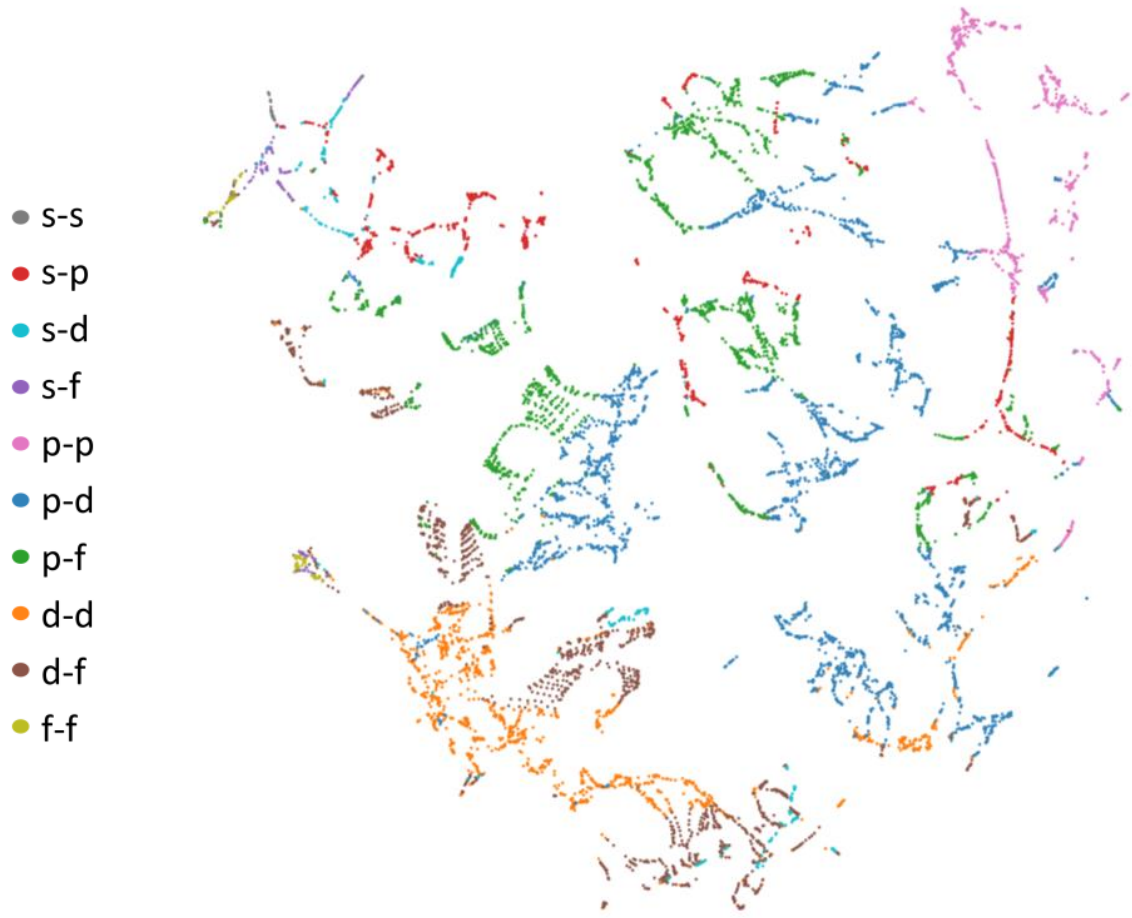
Figure S4. The Mendeleev Number (Villars second ordering) [5] - This follows a similar ordering to the previous going from left to right, bottom to top through the periodic table. As such many regions can be seen to fall in a distorted locality to those of the previous plot.

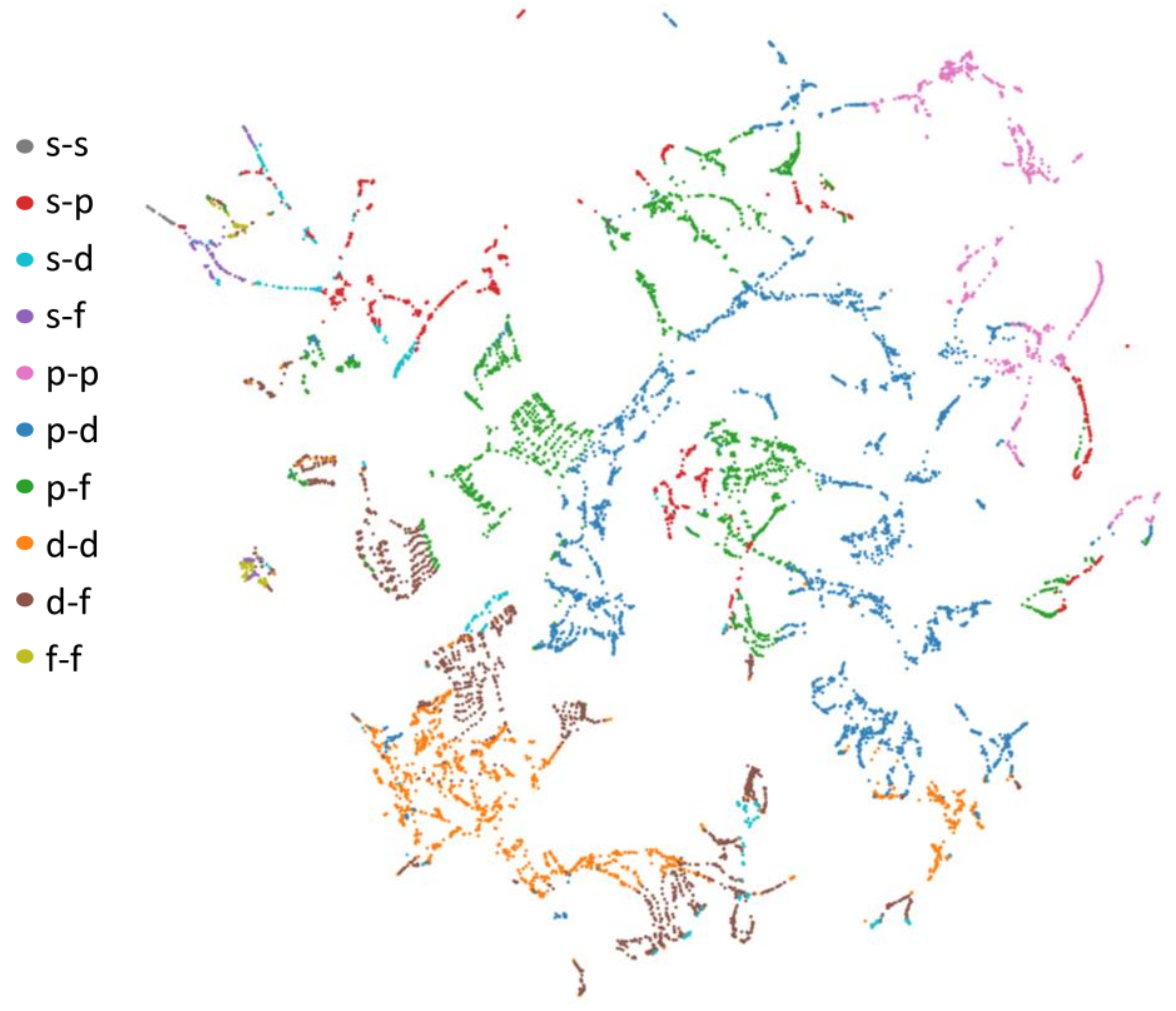

Figure S5. The Mendeleev Number (Villars third ordering) [5] - Here the periodic table is numbered from right to left, top to bottom. Being a reversal of the previous scale, it is unsurprising that this leads to a nearly identical embedding.
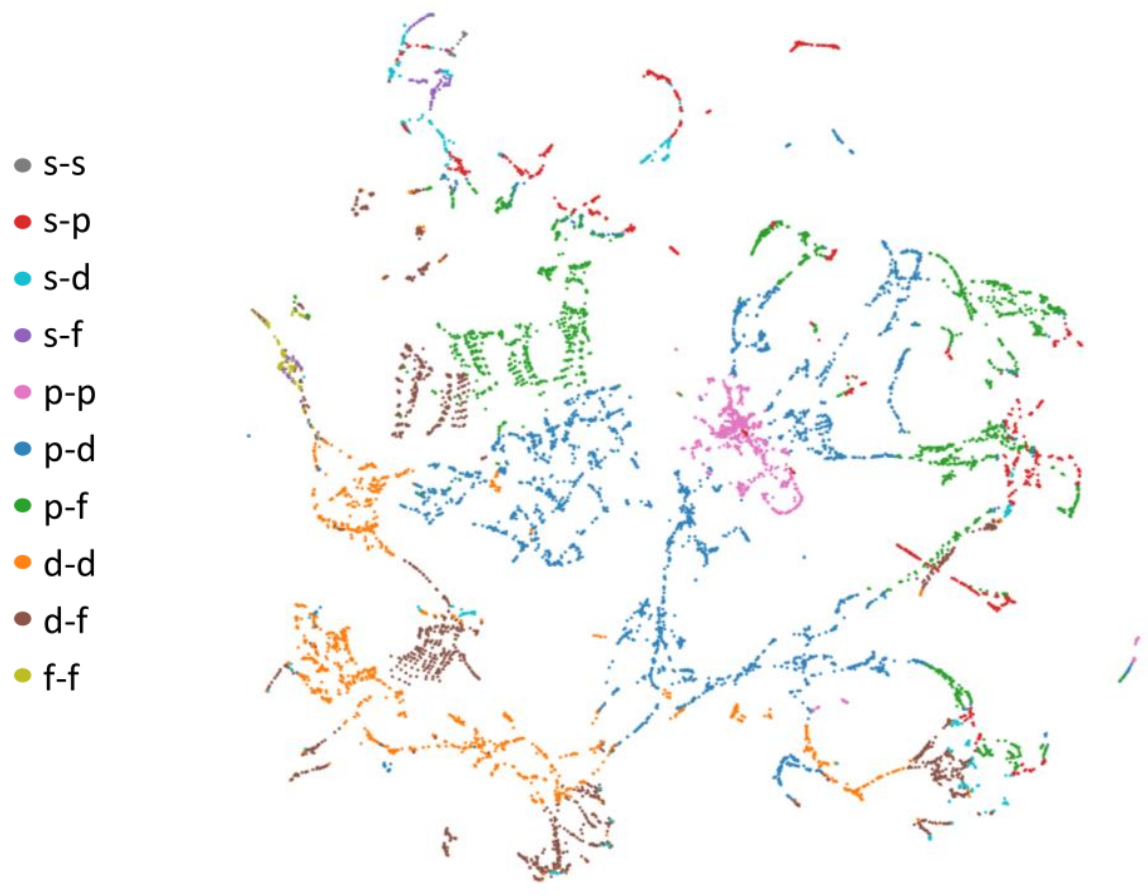
Figure S6. The Mendeleev Number (Villars fourth ordering) [5] - The final ordering (being a reversal of the first) in this instance has gathered the p-p bonded compositions in the center of the embedding, but in most other respects is similar to the previous plots. The migration of these cluster labels may be explained by the stochastic nature of the UMAP algorithm.

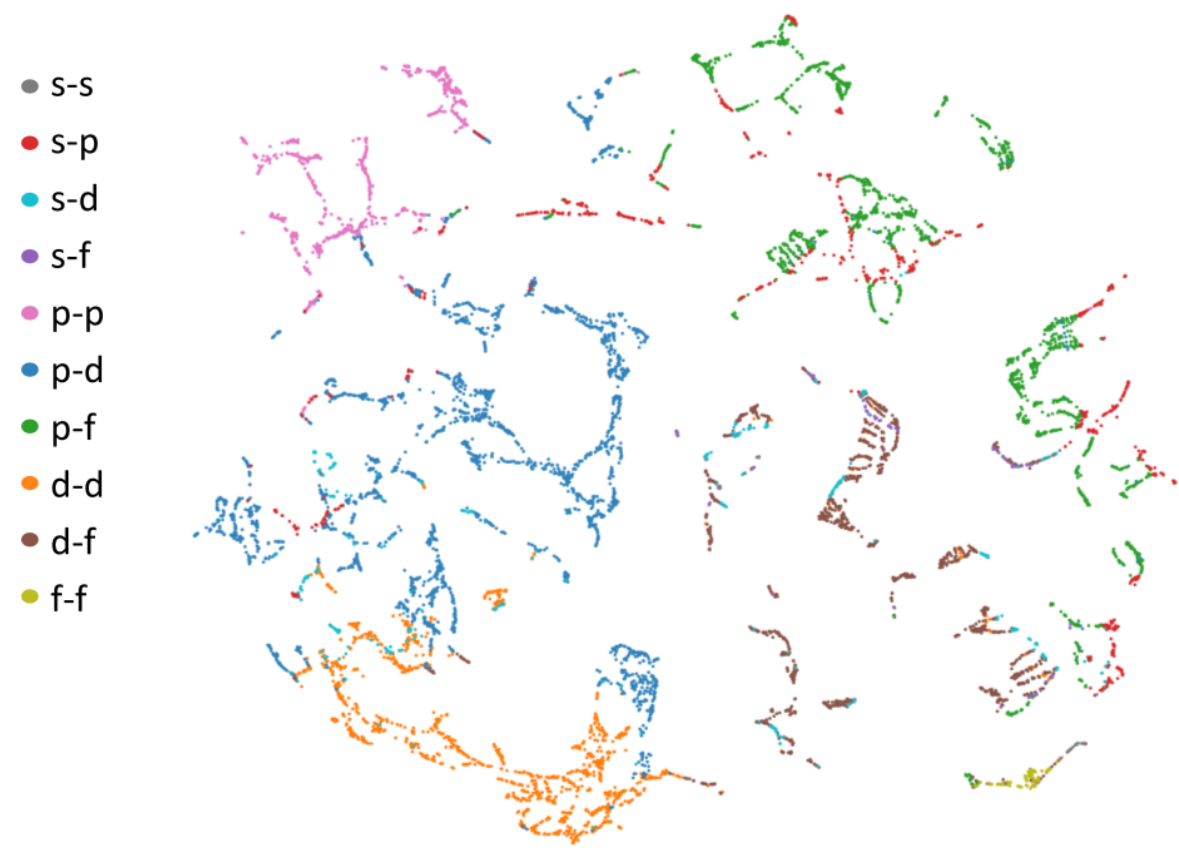

Figure S7. The Modified Pettifor Number [6] - With the modified Pettifor scale we see a purer separation of d-d, p-d, and p-f clusters compared to the previous Mendeleev numbers, and marginally cleaner separation of the d-f compounds compared to the Pettifor scale. Clusters with s-p and $\mathrm{p}$-f compositions show to be difficult to separate with each of these scales.

- S-S

- $s-p$

- $s-d$

- s-f

- $p-p$

- $p-d$

- $p-f$

- d-d

- d-f

- $f-f$
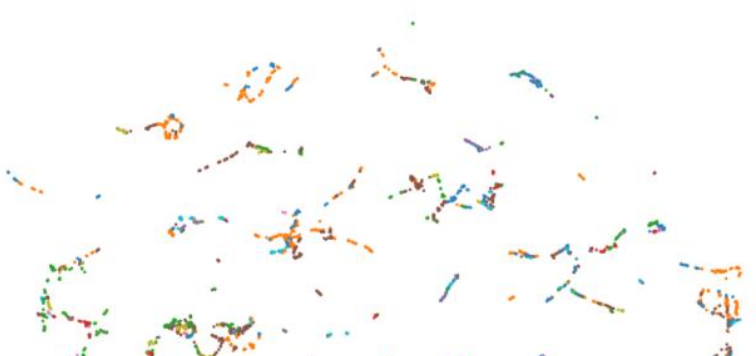

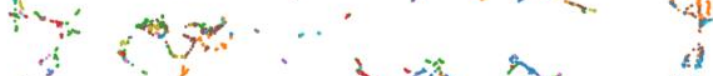
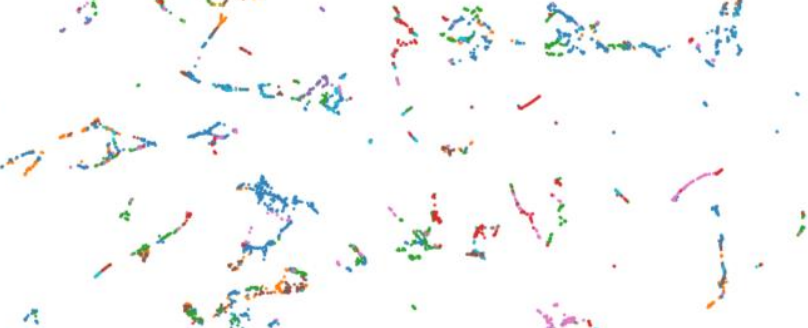

$n$

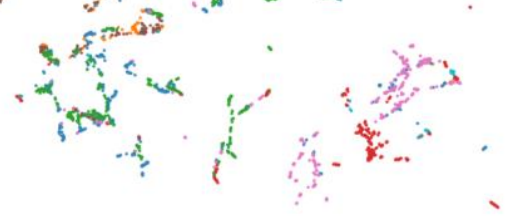


Figure S8. Magpie Descriptors (under the Euclidean metric)[7] - Here we see very little separation into regions of the periodic table with only the p-p compounds showing any strict locality to one another. These may follow better trends in physical properties, however this is not possible to verify with this unlabeled dataset.

\section{DBSCAN analysis of binary compounds}

With 12, 623 reported binary compounds reported in the ICSD the problem of organizing and categorizing these such that the families follow chemical reasoning is a tremendous task which is well suited to machine learning (ML). The application of the EMD to compositions gives us a reasoned measure of similarity between compounds, suitable for clustering. Unfortunately, we may not use traditional clustering algorithms in the metric space directly, as the aforementioned negative curvature means that many of the Euclidean assumptions made by these algorithms, leads to nonsensical decision boundaries in the metric space. The application of the UMAP algorithm is therefore essential, and allows us to condense these relationships into 2-dimensional Euclidean coordinates which maintain appropriate local density whilst distancing from dissimilar neighbors.

Density based spatial clustering applications with noise (DBSCAN) is a popular clustering algorithm which is well suited to the embeddings produced by UMAP. At a high level, these labels are assigned by calculating the distance between all points, and any which are close by to one another are simply labelled as being within the same cluster. A distance threshold, Eps is assigned, and two points will be considered to not share a label if they are further apart than this. DBSCAN with an Eps-neighborhood value of 0.63 on the UMAP embedded points initially creates 39 cluster labels, which when outliers of few compositions have been discarded, gives 26 core clusters.

These primary clusters are of small enough size that we may draw a reasonable qualitative classification, however several contain hundreds of compounds which may not immediately possess a trend which is discernable to the human chemist. Here follows an overview of each of the 26 clusters and the relevant chemical trends. In many of the larger clusters there is simply too much information for text to be an efficient method of communication, and broader descriptions must be given. For ease of reference each cluster is projected to a $4 \times 4$ grid, and the corresponding grid references displayed in boldface. Global $x$ and $y$ intercepts have been overlaid, which due to the neighborhood preservation given to us by UMAP, gives a weak equivalence to the EMD locally. The interested reader may find the full projection online at www.elmd.io/plots/binaries, where an interactive version can be further explored.

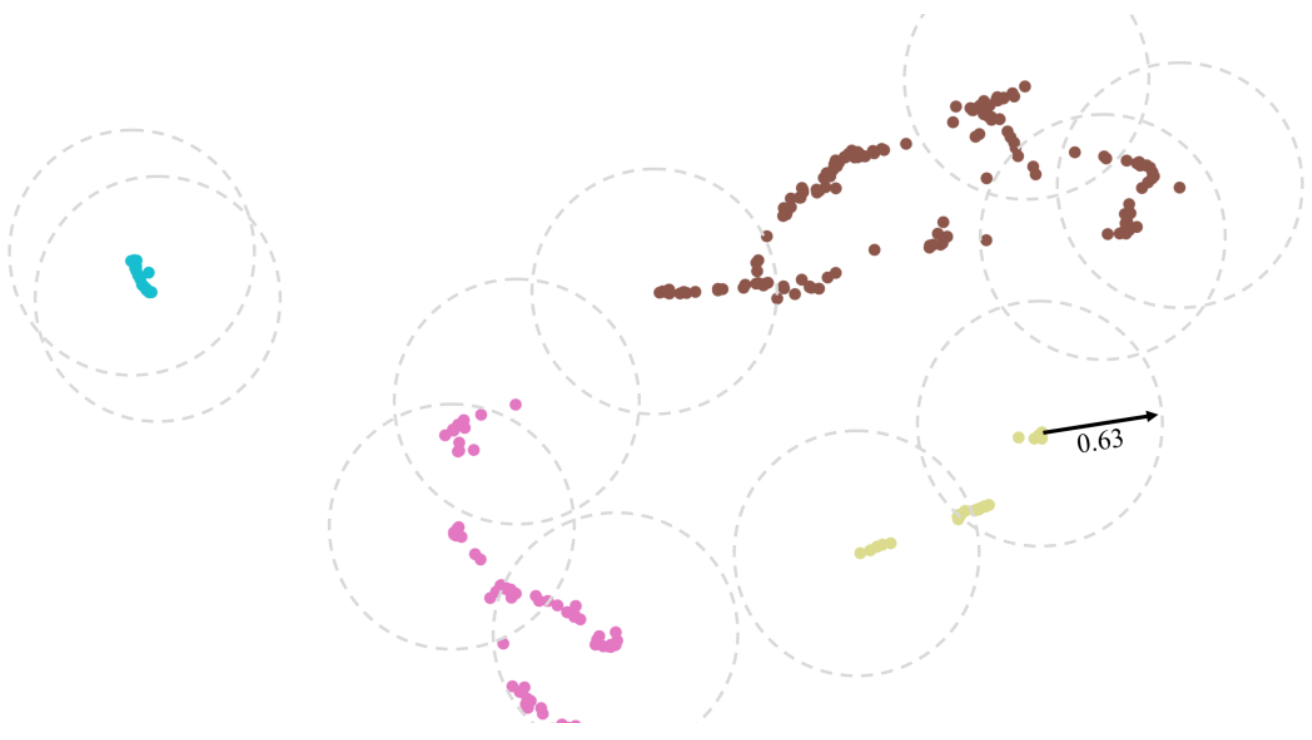

Figure S9. Clusters 14-17 of the binary compounds with disks overlaid on a selection of the cluster boundary points, to highlight the separation of these clusters from one another. In this example, there is an argument that due to their proximities and chemical similarities, clusters 14-16 could be considered to be the same cluster. If we increase the neighborhood radius further to accommodate for this however, we see that major clusters elsewhere in the plot begin to merge also. For this embedding we have found that an Eps value of 0.63 has given good qualitative cluster labels overall, but the choice of this value will be entirely dependent on the dataset. 
Table 1: The count and chemical labels present in each of the clusters assigned by DBSCAN with an epsilon=0.63 on the 12,623 binary compositions in the ICSD (2017). DBSCAN was applied to the points obtained from UMAP on the binary compositions, reduced to two dimensions. The inter-compound EMD of each of these clusters was calculated, with mean, meximum, median, standard deviation, and local correlation of Euclidean distances with the corresponding EMD.

\begin{tabular}{|c|c|c|c|c|c|c|}
\hline Label & Count & Valence Bonds & Mean & Median & $\operatorname{Max}$ & $\begin{array}{l}\text { Standard } \\
\text { Deviation }\end{array}$ \\
\hline Globally & 12623 & & 23.09 & 21.68 & 101.16 & 11.56 \\
\hline 0 & 843 & $\mathrm{p}-\mathrm{p}$ & 6.18 & 6.17 & 16.57 & 2.81 \\
\hline 1 & 335 & p-p, d-p & 5.44 & 5.14 & 15.17 & 2.76 \\
\hline 2 & 262 & d-d, d-p & 6.07 & 5.83 & 19.25 & 2.98 \\
\hline 3 & 295 & $s-p, f-p$ & 5.94 & 5.74 & 17.03 & 2.96 \\
\hline 4 & 516 & $f-p$ & 8.59 & 8.58 & 24.06 & 3.89 \\
\hline 5 & 40 & $s-p$ & 4.84 & 4.44 & 11.96 & 3.24 \\
\hline 6 & 914 & $s-p, f-p$ & 10.28 & 9.83 & 33.93 & 4.87 \\
\hline 7 & 223 & $f-p$ & 4.84 & 4.58 & 14.71 & 2.74 \\
\hline 8 & 830 & d-f, s-p, f-p & 11.21 & 10.50 & 49.75 & 5.96 \\
\hline 9 & 290 & $f-p, s-p$ & 9.05 & 8.83 & 23.86 & 4.61 \\
\hline 10 & 250 & f-d, s-s & 10.39 & 8.60 & 41.59 & 7.56 \\
\hline 11 & 688 & f-d, s-d, s-p & 12.11 & 11.58 & 35.15 & 5.81 \\
\hline 12 & 204 & $f-d, f-p, f-s$ & 7.56 & 6.25 & 26.50 & 5.29 \\
\hline 13 & 496 & $f-d$ & 7.23 & 7.00 & 22.00 & 3.58 \\
\hline 14 & 222 & f-d, s-d & 4.42 & 4.32 & 13.71 & 2.11 \\
\hline 15 & 32 & s-s & 2.10 & 2.21 & 5.57 & 1.36 \\
\hline 16 & 27 & s-f & 0.65 & 3.05 & 1.86 & 0.46 \\
\hline 17 & 178 & $\mathrm{~d}-\mathrm{d}$ & 3.11 & 0.57 & 8.48 & 1.64 \\
\hline 18 & 40 & f-d & 1.41 & 1.28 & 4.90 & 0.96 \\
\hline 19 & 425 & f-d & 5.58 & 5.55 & 14.67 & 2.41 \\
\hline 20 & 2729 & d-d, d-p, d-f & 10.20 & 9.71 & 48.30 & 5.25 \\
\hline 21 & 90 & d-f & 2.43 & 2.43 & 6.40 & 1.43 \\
\hline 22 & 64 & $d-p$ & 3.01 & 2.92 & 7.33 & 1.60 \\
\hline 23 & 931 & d-s & 6.09 & 5.40 & 22.40 & 3.49 \\
\hline 24 & 1411 & $d-p$ & 10.84 & 10.62 & 30.69 & 4.84 \\
\hline 25 & 288 & $d-p, f-p$ & 3.81 & 3.52 & 16.00 & 2.30 \\
\hline Average & 485.5 & & 6.28 & 5.95 & 20.45 & 3.34 \\
\hline
\end{tabular}



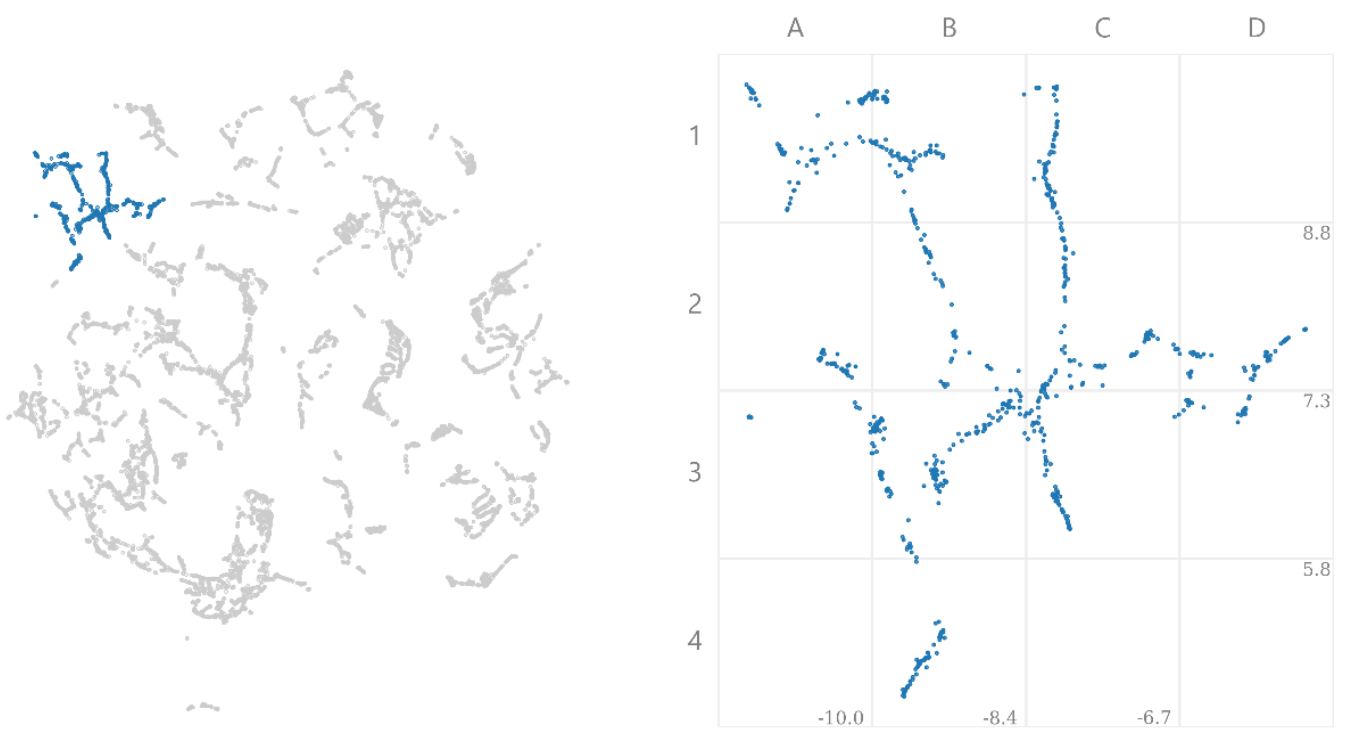

Label 0

Consisting entirely of p-block binary compounds. Towards the center of the plot at $\mathbf{B 3}$ we see compounds with a nearly equal $A B$ ratio with some variance. Each of the trend lines from the center lead towards a higher ion doping in a reasonably smooth fashion. It can be seen that each branch has a reasonably strong separation into different chemical families amongst the p-block elements. In the upper left following the trend from $\mathbf{A} 1$ to $\mathbf{B 2}$ we find two non-metals (non-halogens) in the ratio $A B_{2} \rightarrow A B_{9}$. Non-metals bonded to p-block metals are found in $\mathbf{A} 2$ to $\mathbf{B} 4$, from $A B_{9} \rightarrow A_{2} B_{3}$, with the trend to the center leading to binary non-metal compositions. Transition metals bonded to a pnictogen element in high doping are found in $\mathbf{C 3}$. The region covered by $\mathbf{C 1}$ to $\mathbf{D 2}$ is majority B and $\mathrm{C}$ containing compounds, which increase in these elements concentration to the rightmost tip to almost pure $\mathrm{B}$ with $\mathrm{V}_{3} \mathrm{~B}_{497}$.

Chemical Families:

- Upper Left: Two p-block non-metals, non-halogens

- Lower Left: p-block + p-block

- Lower Middle: Transition metal + pnictogen

- Upper Middle: Group IV + halogen

- Right: p-block metal + highly doped B/C

Composition Trends:

- Upper Left: $A B_{2} \rightarrow A B_{9}$

- Lower Left: $A B_{9} \rightarrow A_{2} B_{3}$

- Lower Middle: $A B_{3} \rightarrow A B_{4}$

- Upper Middle: $A B \rightarrow A_{4} B_{121}$

- Right: $A B \rightarrow A_{2} B_{123}$

Chemical Trends:

- Upper Left: $B$ ion increasing in atomic weight down chalcogen group. Partner ion follows metalloids plus carbon.

- Lower Left: Both ions increase in electronegativity from bottom to top.

- Lower Middle: p-block metal + non-metal, non-metal trends towards B/C as we move right

- Upper Middle: Carbon concentration dropping as we move up the cluster

- Right: Increase in B/C doping from left to right. Some silicides and germanides present in the center of the cluster. 

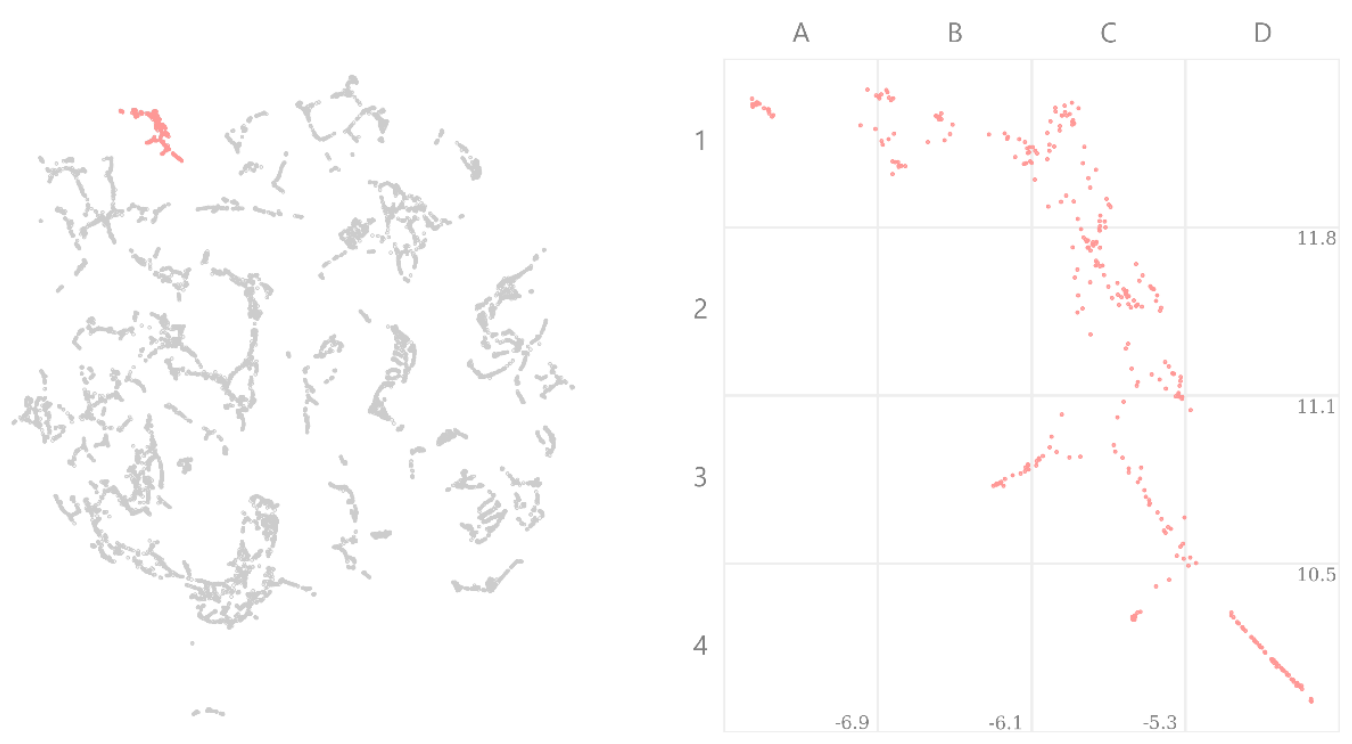

Label 1

An island of Te/Se sulfides found to the left of $\mathbf{A 1}$ leads to Te/Se iodides in B1 before the remaining chalcogen halides from $\mathrm{BiI}$ to $\mathrm{OH}_{2}$ are found in $\mathbf{C 1}$. Moving down the larger cluster we find the $A$ ion moving through the metal-halides from $\mathrm{PoF}_{5}$ to $\mathrm{InF}_{3}$ in the general form $A B_{3}$. Reaching the split in $\mathbf{C 3}$, towards the left are $\mathrm{B} / \mathrm{C}$ fluorides and hydrides, from $\mathrm{BF}_{2}$ to $\mathrm{B}_{9} \mathrm{H}_{11}$. Following the branch to the right we see the p-block metal-halides from $\mathrm{GeF}_{2}$ to $\mathrm{PbI}_{2}$, until the lower split which follows boron group-halides to $\mathrm{Ga}_{2} \mathrm{I}_{3}$ in $\mathbf{C 4}$, and transition metal-halides of the form $A B_{2}$ from $\mathrm{HgI}_{2}$ to $\mathrm{PdI}_{2}$ in $\mathbf{D 4}$.

Chemical Families:

- Upper: Chalcogen + halogen

- Middle: Transition metal + halogen

- Lower: Transition metal + halogen

Composition Trends:

- Upper: Large variance of $A B$ ratio

- Middle: Generally of the form $A B_{3}$

- Lower: Trending to the left $A B_{2} \rightarrow A B$, to the right trends $A_{3} B_{17} \rightarrow A B_{2}$

Chemical Trends:

- Upper: From chalcogen + chalcogen pairs to chalcogen + halogen pairs at the top.

- Middle: $A$ ion decreases in electronegativity towards the carbon group moving down

- Lower: To the left, boron/carbon halides. As we move down, $A$ ion decreases in electronegativity through the p-block metals. 

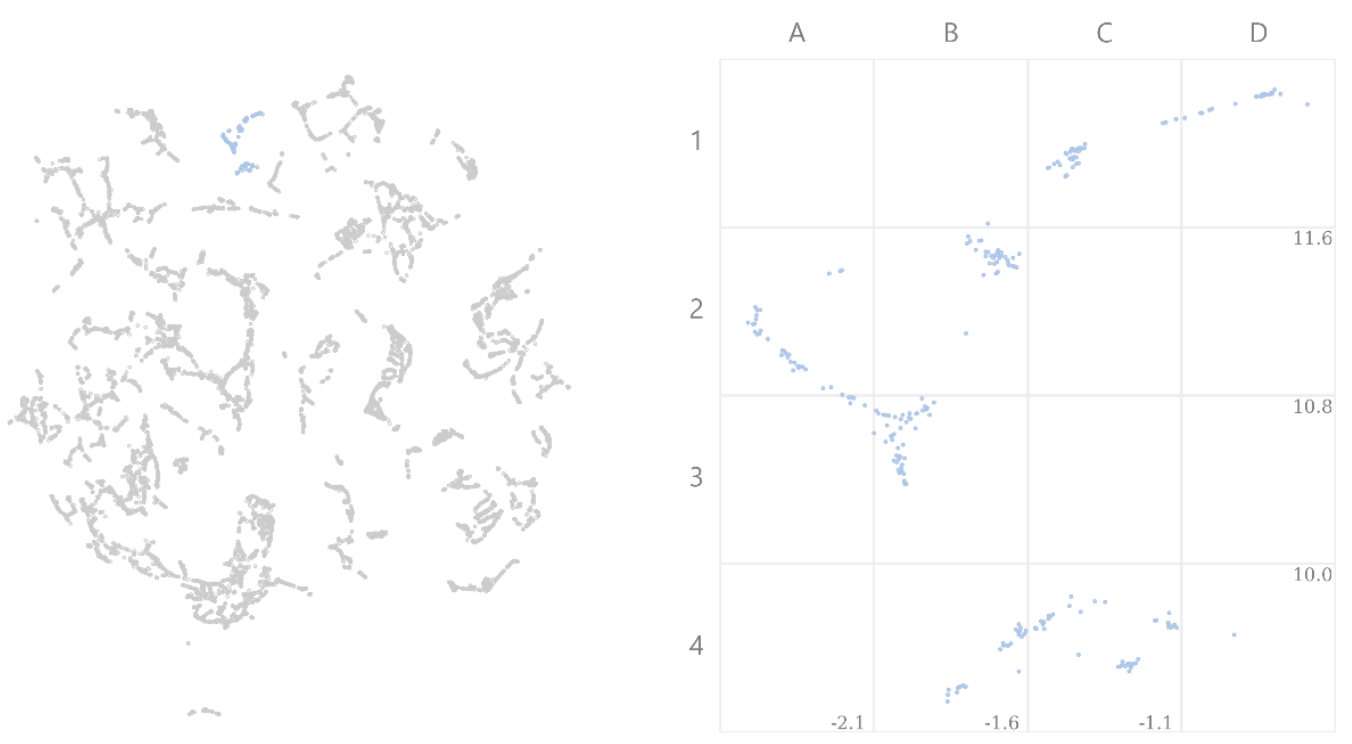

Label 2

Here we see a collection of metal-halides, with metal-chalcogenides in B4-C4, which all possess a strong $B$ doping of 70-83\% concentration. The initial trendline through $\mathrm{D} 1$ sees f-block metals bonded to $\mathrm{F}$ and $\mathrm{H}$ of the form $A B_{4}$. Moving down we see three successive clusters with a smoothly increasing $A$ ratio from $\mathbf{C 1}$ to $\mathbf{B 3}$ from $\mathrm{OsF}_{5}$ to $\mathrm{Ta}_{3} \mathrm{Br}_{7}$, with fewer elemental trends through the periodic table found in this region to accommodate for this smooth change in ratio. The trend from $\mathbf{A 2}$ to $\mathbf{B 3}$ follows transition metalhalides of the form $A B_{3}$ with the metal decreasing in electronegativity as we move down. The final cluster of points in B4-C4 follows transition metal chalcogenides from $\mathrm{V}_{3} \mathrm{O}_{7}$ to $\mathrm{UTe}_{5}$.

Chemical Families:

- Upper: f-block metal + halogen

- Middle: Transition metal + halogen

- Lower: Transition metal + chalcogen

Composition Trends:

- Upper: $A B_{5} \rightarrow A B_{6}$

- Middle: $A B_{3} \rightarrow A B_{4}$

- Lower: $A_{3} B_{7} \rightarrow A B_{5}$

Chemical Trends:

- Upper: From chalcogen + chalcogen pairs to chalcogen + halogen pairs at the top.

- Middle: $A$ ion decreases in electronegativity towards the carbon group moving down

- Lower: To the left, boron/carbon halides. As we move down, $A$ ion decreases in electronegativity through the p-block metals towards transition metal halides at the very bottom. 

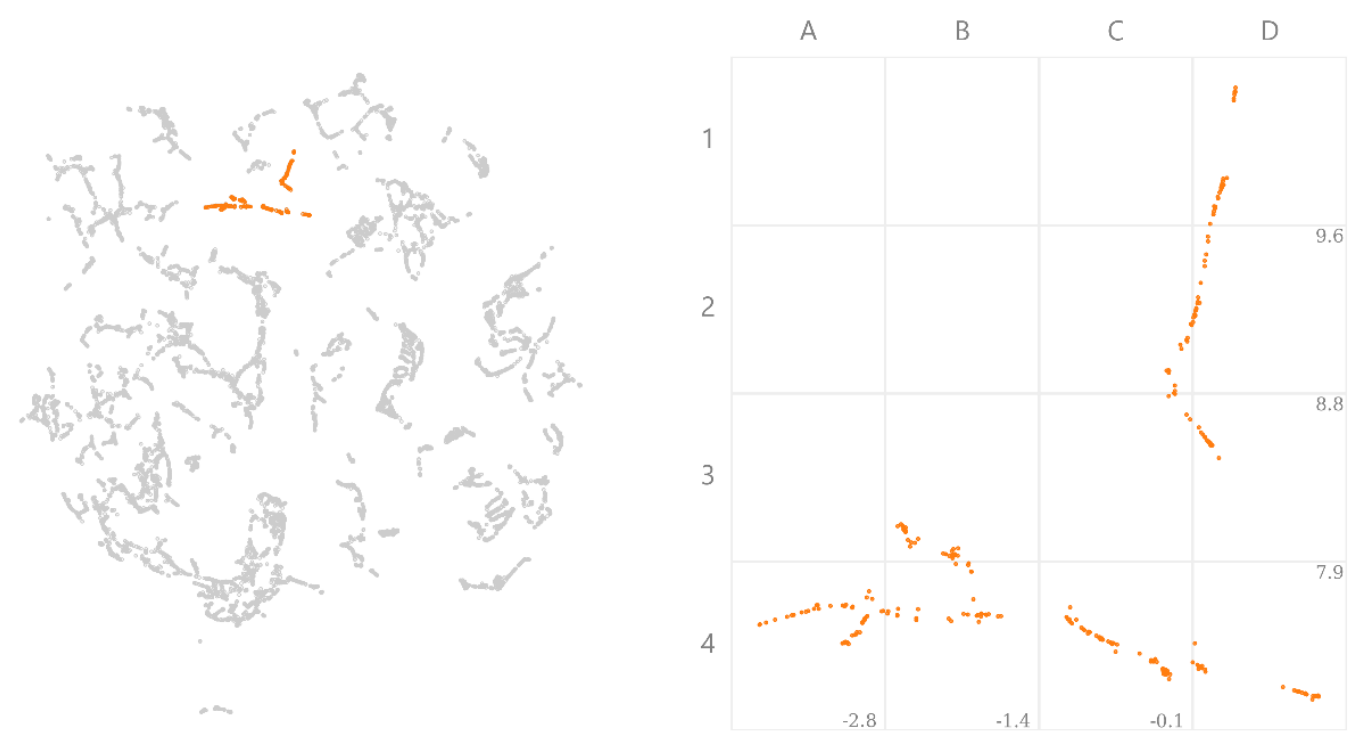

Label 3

The leftmost points of $\mathbf{A 4}$, with $\mathrm{Na}_{0.021} \mathrm{Si}_{0.979}$, follow the high $B$ doping that can be found at the rightmost tip of cluster 0 . This doping falls, but remains high as we follow the remaining alkali/alkaline metals along row $\mathbf{4}$, mostly as silicides but with some other carbon group $B$ ions, to $\mathrm{SrSn}_{4}$. The lower and upper branches along this trend, contain transition/f-block metal borides (A4) and carbides (B3) respectively. The line from $\mathbf{D 1}$ to $\mathbf{D 3}$ tends to follow f-block metal-carbon group compounds, from $\mathrm{Ba}_{0.176} \mathrm{P}_{0.824}$ to $\mathrm{Nd}_{0.182} \mathrm{Ge}_{0.818}$.

Chemical Families:

- Upper: Lanthanide + p-block

- Left: Alkali/alkaline/lanthanides + p-block

- Center: Lanthanide borides

Composition Trends:

- Upper: $A B_{7} \rightarrow A_{3} B_{17}$

- Left: $A_{0.021} B_{0.979} \rightarrow A B_{4}$

- Center: $A B_{4}$

Chemical Trends:

- Upper: Mostly lightly doped lanthanide carbides and borides, with greater variation at the top of the cluster, phosphides towards the center.

- Left: Mostly silicides, with a little variation amongst the metalloids combined with an alkali metal, with some alkaline metal/lanthanides.

- Center: Borides of the form $A B_{4}$ paired with an alkaline metal to the left, lanthanides to the middle, and light actinides in the far right cluster. 

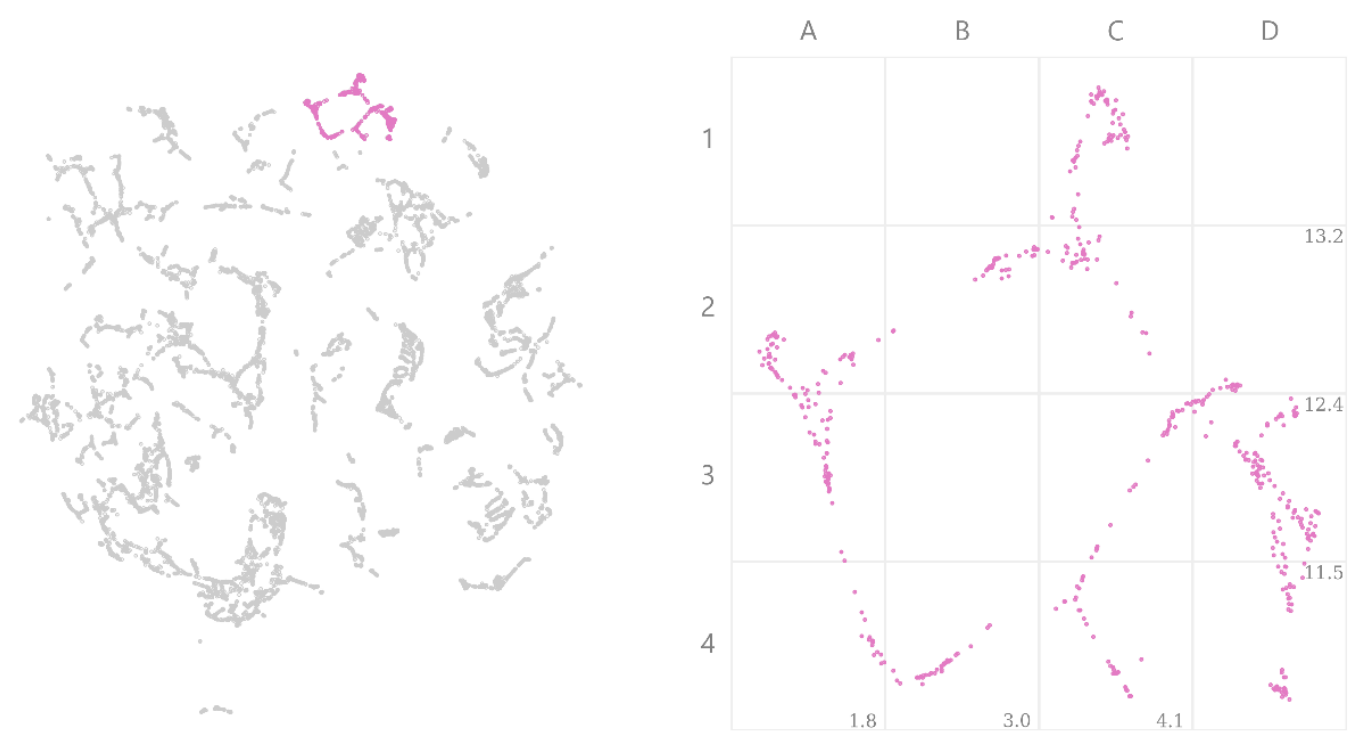

Label 4

This cluster possesses one of the clearest ring structures in the projection. Entirely consisting of ionic compounds (lanthanides and actinides bonded to a chalcogen/halogen $B$ ion) this gives an interesting series of feasible substitutions and minor changes in stoichiometry, which will eventually return to the same compound. The upper and lower left regions of the ring are of the form $A B_{3}$ which smoothly trend to $A B_{2}$ compounds on the right of the cycle and the lanthanide-chalogenides found in D3-D4.

Chemical Families:

- Left: Actinide/lanthanide/alkali + halogen/chalcogen

- Upper: Lanthanide/s-block + halogen

- Central and Right: Lanthanide/actinide + chalcogen

Composition Trends:

- Left: $A B_{3}$

- Upper Cluster: $A B_{3} \rightarrow A B_{2}$, trace $A_{2} B$ at top

- Central and Right: $A B_{2} \rightarrow A_{0.433} B_{0.567}$

Chemical Trends:

- Left: Top left we have $A$ ions as actinides in the form $A B_{3}$ which transition through the lanthanides, then alkaline metals combined with a halogen. After this, a $B$ chalcogen, with $A$ moving back through the alkali, lanthanide and actinides towards the center of the cluster.

- Upper: Starting with a lanthanide and halogen on the left of this region, trending to an increase in $B$ ratio as we go towards the center of the cluster. This splits off into the s-block metals towards the top of the cluster in the form $A_{2} B$

- Central and Right: Follows the trend of the left hand cluster in composition and makeup, splitting into two branches, for lanthanides and actinides, each paired with a chalcogen, of the form $A_{0.029} B_{0.71}$ and $A B_{2}$ respectively. As the upper cluster merges with the central cluster the $A$ ratio continues to drop to the bottom right, with exclusively lanthanide $A$ ion with a chalcogen 

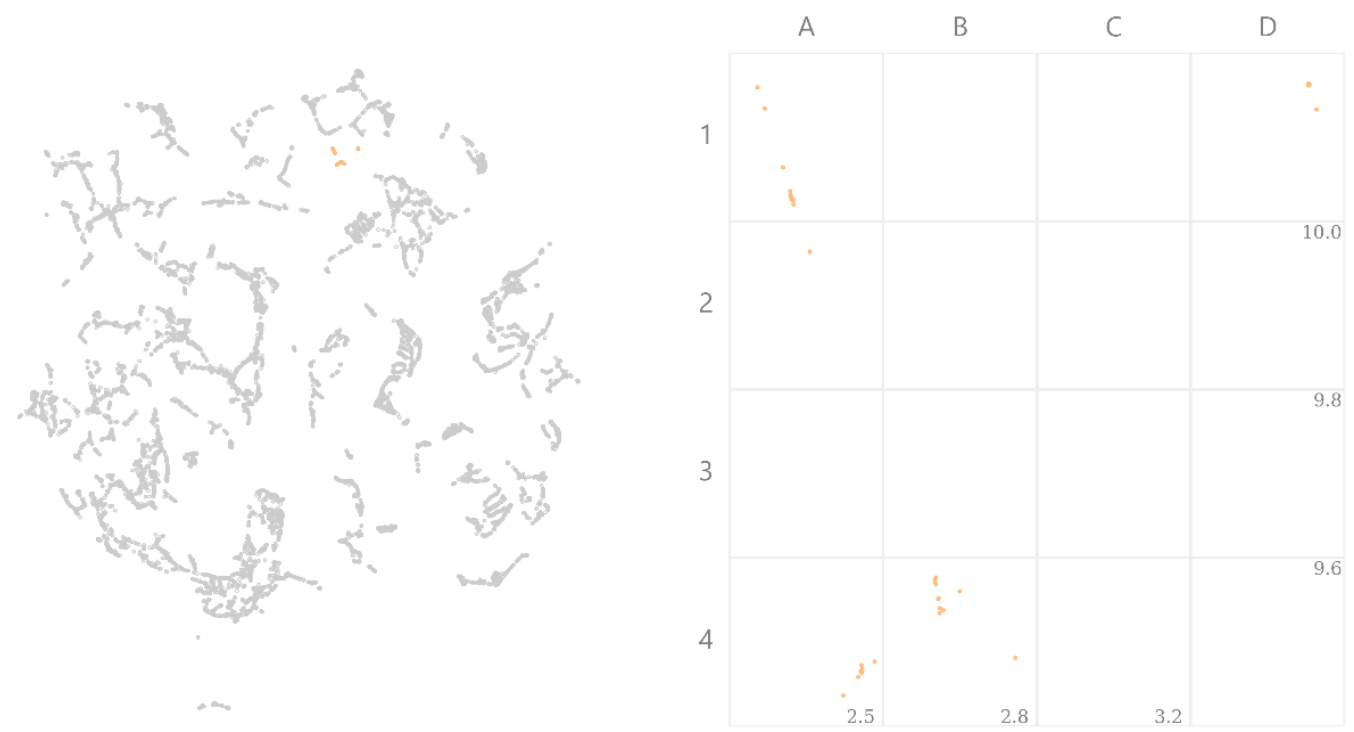

\section{Label 5}

As with the previous family these are entirely ionic compounds, being an s-block metal combined with a p-block element of the form $A_{0.286} B_{0.714}$ to $A_{0.214} B_{0.786}$, with $B$ ions being $\mathrm{Bi} / \mathrm{Sb}(\mathbf{A 1}), \mathrm{P} / \mathrm{N}(\mathbf{A 2}-\mathbf{B} 4), \mathrm{Se} / \mathrm{Te}(\mathbf{D 4})$. A ring structure can be loosely interpreted when viewed in conjunction with the previous plot, however due to the fewer number of reported compositions there are larger jumps in makeup and stoichiometry when we attempt to follow a cycle. 

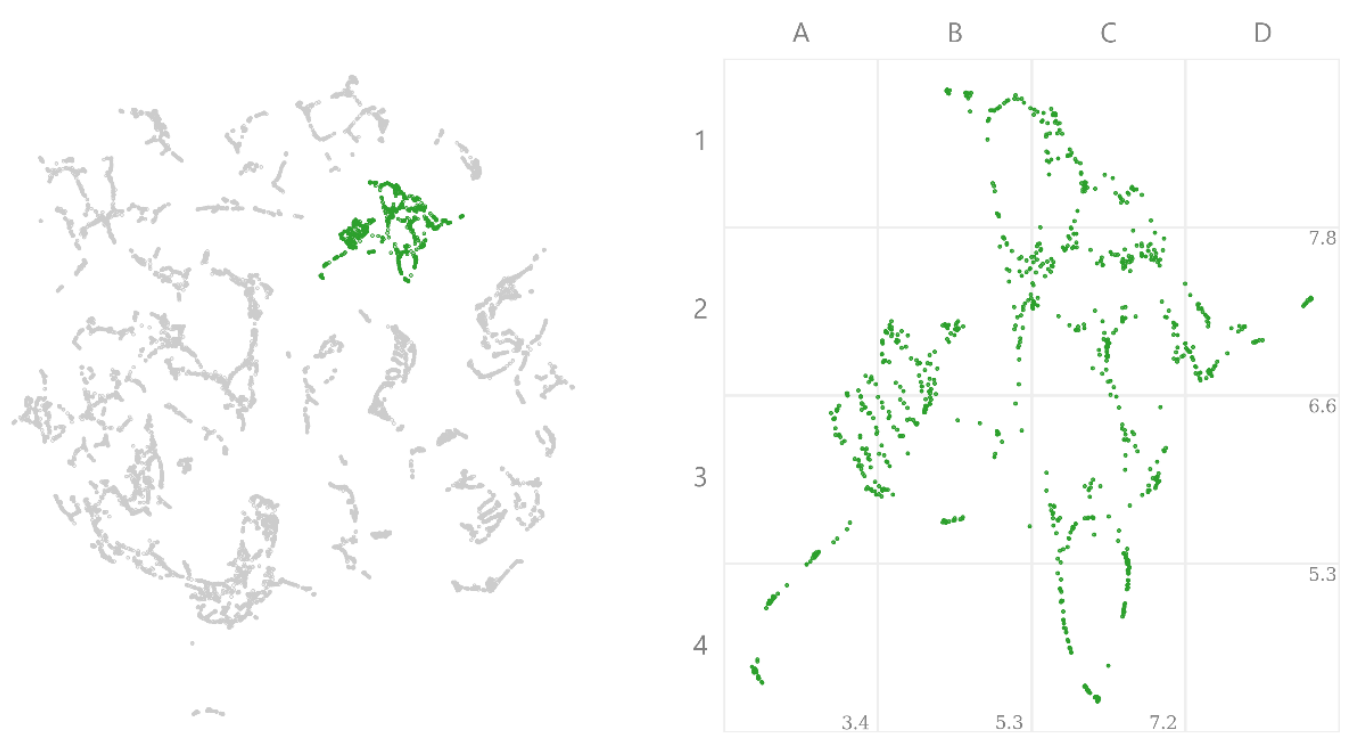

Label 6

Here we see a larger collection of ionically bonded compounds which possesses both cycles of similar compositions, as well as approximate parallel lines found from A2-B3. Each of these lines represent compositions of the form $A B_{3}$, with each distinct line containing the same $B$ ion. Across the entire plot from lower leftmost point to the far right there is a consistent transition in $A B$ ratio from $\mathrm{PuAl}_{4}$ to $\mathrm{Rb}_{2} \mathrm{O}_{3}$. From top to bottom there is a general decrease in $B$ ion electronegativity from As to In, as $A$ ions go through the s-block to the f-block metals.

Chemical Families:

- Left: Lanthanide $A$ ion $+B$ ion from the heavier boron group elements.

- Central: At the top contains alkali metals and alkaline metal $A$ ions, with a pnictogen $B$ ion. Towards the middle, more alkaline metal and lanthanide $A$ ions with the $B$ ion trending towards the $\mathrm{C} / \mathrm{N} / \mathrm{P}$ groups.

- Lower: Boron group + lanthanides/actinides. These trends follow to the right, increasing in $A$ ion ratio.

Composition Trends:

- Left: $A B_{4} \rightarrow A B_{3}$

- Central: $A B_{2}$ down the center of the cluster $\rightarrow A_{2} B_{3}$ to the right

- Lower: $A B_{2}$

Chemical Trends:

- Left: $B$ ion ratio increasing from 0.2 to 0.32 to the center of the cluster. Characteristic parallel lines for $A B_{3}$ in the middle of the left hand, with each line consisting of a single boron group metal for each $B$ ion. $A$ ions trend from lanthanides in the far left, splitting into alkali/alkaline metal, lanthanide, and actinide in the center. These trends follow to the right.

- Central: As the trend moves further down the cluster, the $B$ ion moves through the heavier carbon group, which tend to pair with an alkali or alkaline metal in the center. $B$ ions tend to start in the boron group, trending to the right of the periodic table in the upper center of the cluster.

- Lower: $B$ ion heavier carbon/boron group with heavier lanthanides 

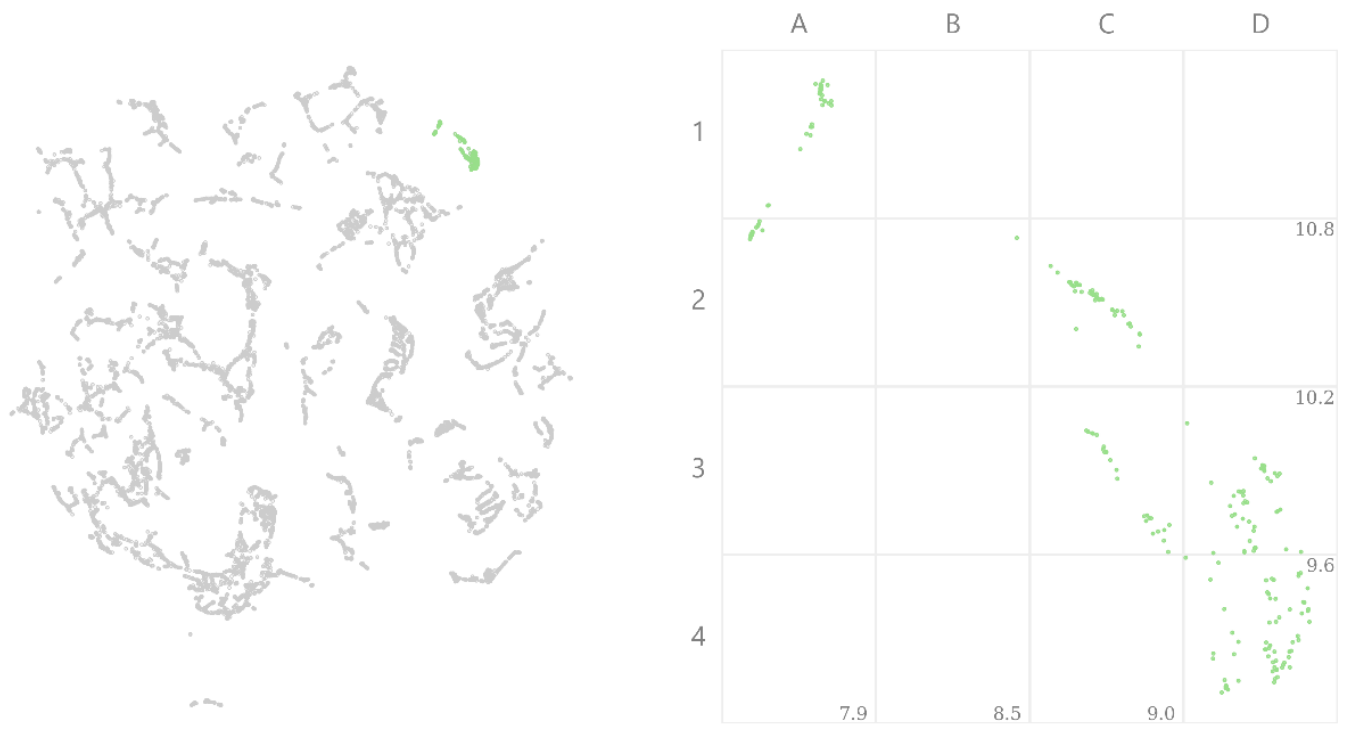

Label 7

The final cluster of ionic compounds for the most part continues the $A_{2} B_{3}$ ratio from the previous cluster, for f-block metalchalcogenides, found in $\mathbf{D 4}$, with $\mathrm{Eu}_{2} \mathrm{O}_{3}$ found at the bottom most point. There is some separation into respective $B$ ions through the chalcogen family as we follow the plot through each cluster up, with the inner crescent (C3) being of the separate ratio, $A_{0.429} B_{0.571}$.

Chemical Families:

- Left: A collection of actinides and chalcogens with increased chalcogen ratio as we move down the cluster of the form $A_{2} B_{3}$, where $A$ is typically $\mathrm{U} / \mathrm{Th} / \mathrm{Np} / \mathrm{Am} / \mathrm{Cm}$

- Right: From the upper left to the lower right the $A$ ions follow the trend of the lanthanide series with a chalcogen ion, in small clusters separated by minor variations in ratio.

Composition Trends:

From left to right in two main clusters

- Left: $A_{0.429} B_{0.571} \rightarrow A_{2} B_{3}$

- Right: $A_{0.429} B_{0.571} \rightarrow A_{2} B_{3}$ 

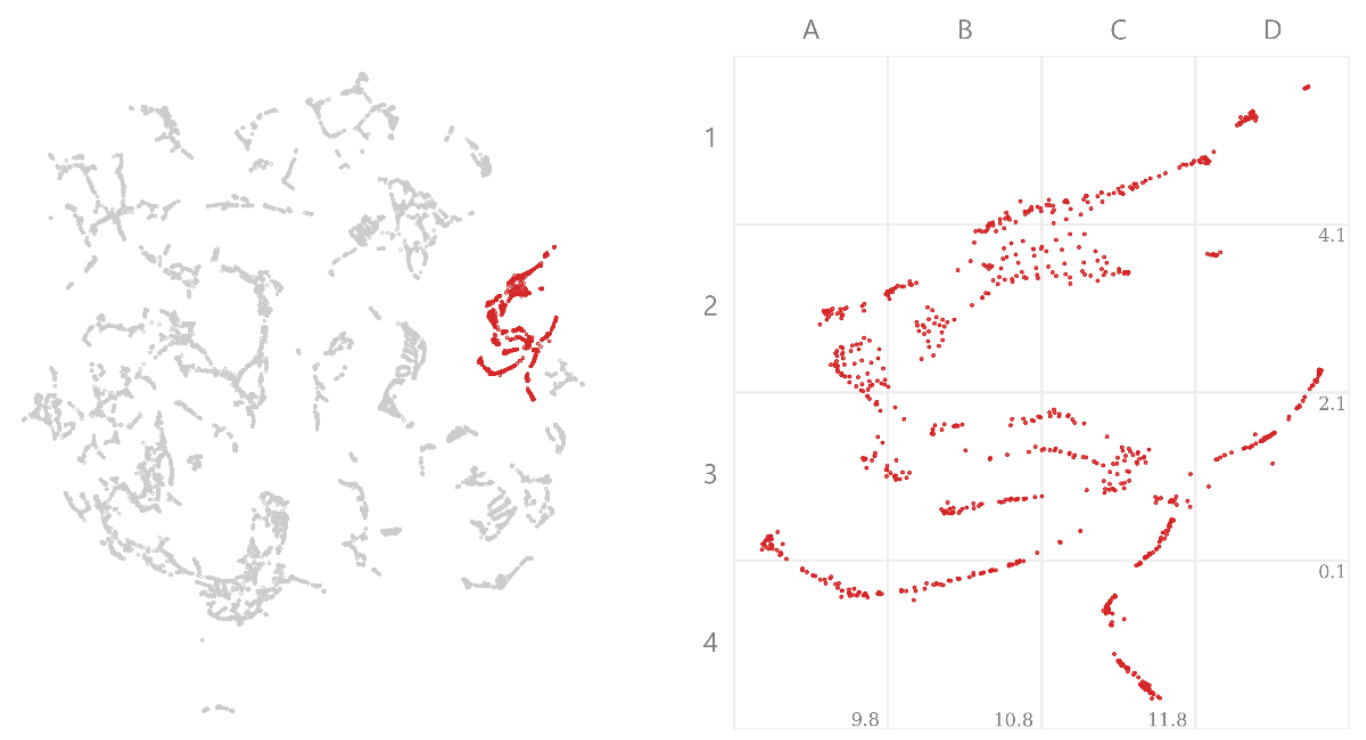

Label 8

The majority of compositions in this cluster are $A B$ compounds barring the lower branch (C4) which follows increasing $A$ ion ratio to $\mathrm{Nd}_{5} \mathrm{Ge}_{3}$. Here we see a reasonably wide variety of metallic elements from the s-block and f-block bonded with a similarly varied selection of p-block elements, reaching to the group 12 metals in the lower left branch. We see some regularity in the upper region where we may find a reasonably complete collection of $A B$ lanthanide-chalcogenides.

Chemical Families:

- Upper: Each of the lines moving up the cluster follow a $B$ ion in the p-block. $A$ ion trends towards the left of the transition block metals.

- Lower: metallic $A$ ion with p-block B ion with

Composition Trends:

- Upper: $A B$

- Lower: $A B$, some trending $A_{5} B_{4} \rightarrow A_{5} B_{3}$ in bottom branch

Chemical Trends:

- Upper: Increased chalcogen ratio as we move down the cluster of the form $A_{2} B_{3}$, where $A$ is typically $\mathrm{U} / \mathrm{Th} / \mathrm{Np} / \mathrm{Am} / \mathrm{Cm}$

- Lower: Each of the arms from the center of this cluster show a classification in either composition or ratio. The center is $A B$ compounds with high electronegativity, upper right contains alkali metals, lower right have divergent ratio from $A B$, bottom left are the remaining $A B$ compounds. Moving up the cluster we see a split into multiple lines, each following a period in decreasing atomic weight as we move up. 

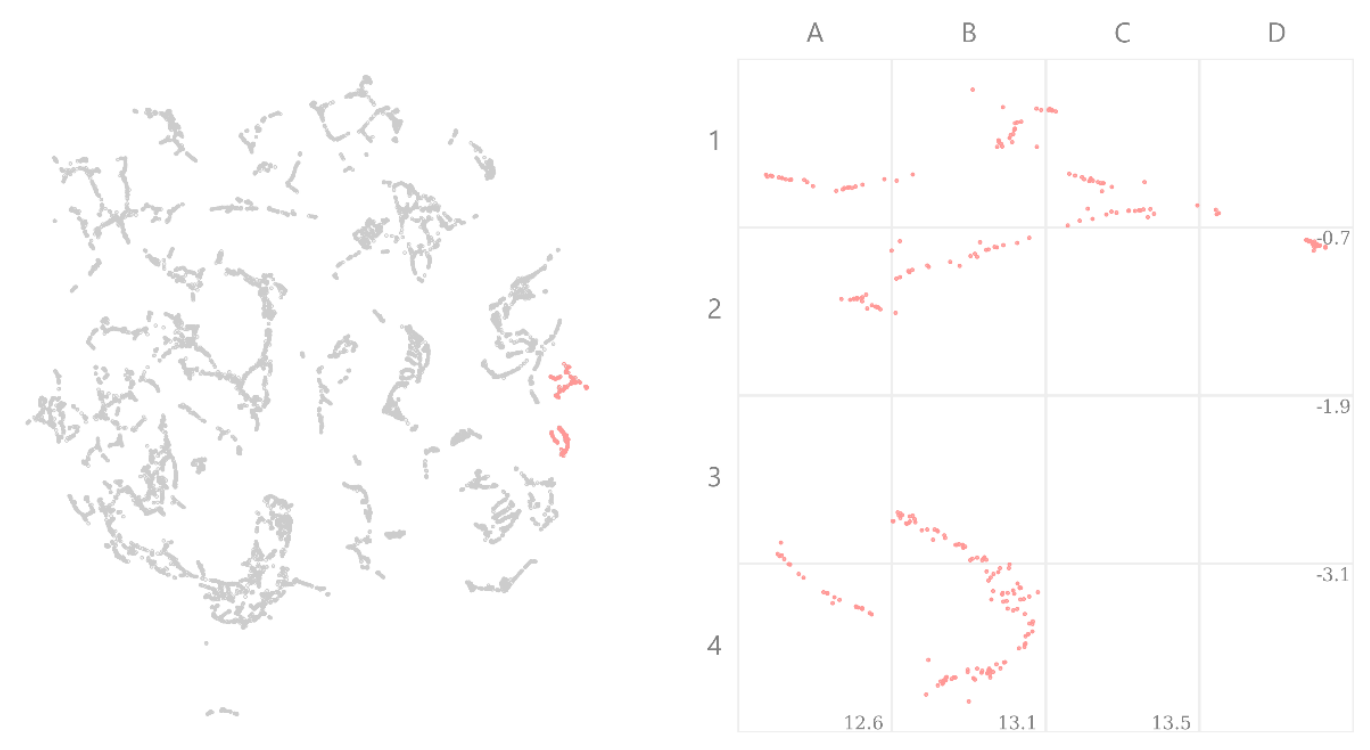

Label 9

Here we follow the increasing $A$ ion ratio from the previous cluster, reaching to $\mathrm{Na}_{2} \mathrm{~S}$ to the far right, with $\mathrm{Sm}_{2} \mathrm{O}$ at the bottom of the plot. We see the typical trend from lanthanides to alkaline metals and alkali metals, repeated for each of the respective compositional ratios found in each cluster, with $\mathrm{La}_{3} \mathrm{Al}_{2}$ at the bottommost tip of $\mathbf{B 4}$, where two DBSCAN clusters have been merged for brevity.

Chemical Families:

- Upper: Lanthanide/alkali/alkaline/actinides + boron/carbon group.

- Lower: Lanthanide/alkali/alkaline $+\mathrm{Al} / \mathrm{Ga} \rightarrow$ p-block metals.

Composition Trends:

- Upper: $A_{4} B_{3} \rightarrow A_{5} B_{3} \rightarrow A_{3} B_{2}$ moving down.

- Lower: $A_{3} B_{2}$ and $A_{3} B_{5}$ in two distinct clusters.

Chemical Trends:

- Upper: No particular chemical trend barring those matching the clusters of ratio.

- Lower: From the upper left the $B$ ion is $\mathrm{Al} / \mathrm{Ga}$, which transitions through the p-block metals in increasing electronegativity as we move down the cluster, trending through lanthanides to alkaline to alkali metal $A$ ions moving down the cluster. 

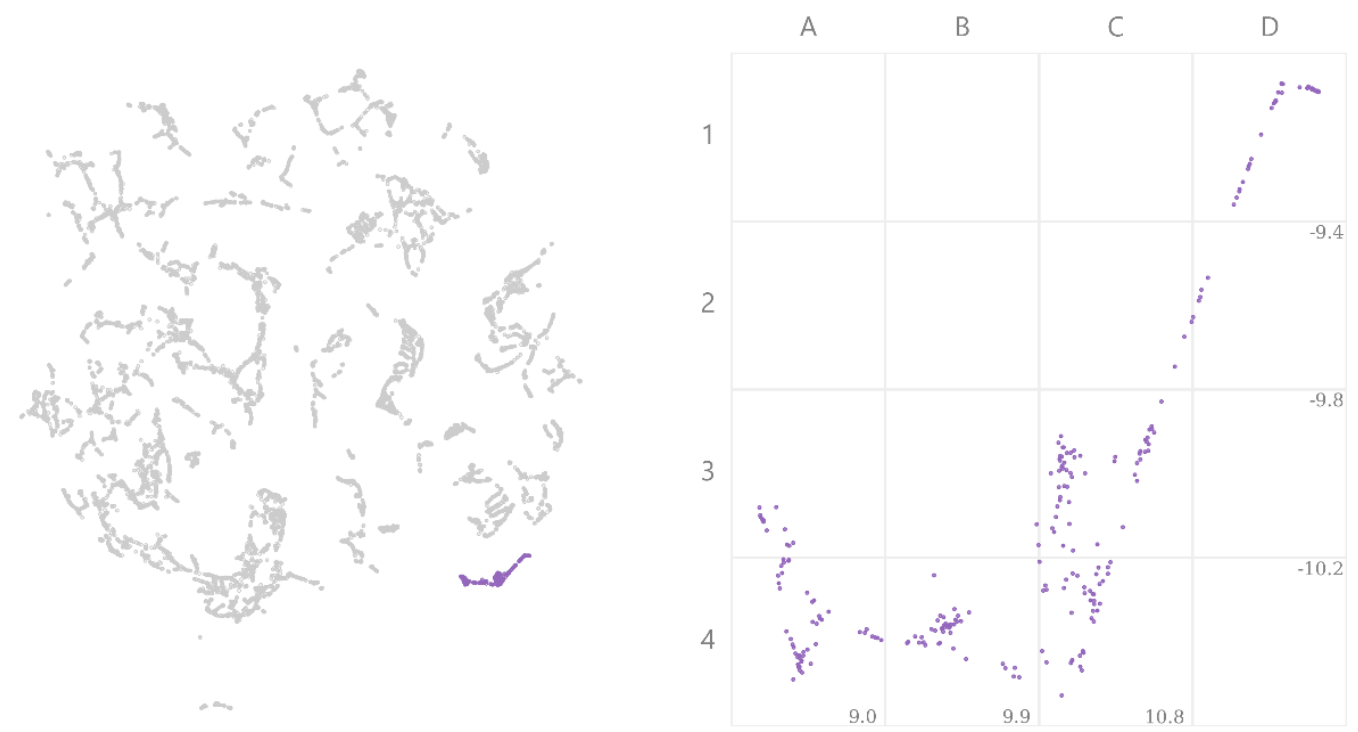

Label 10

The region in $\mathbf{C 3}$ contains the reported binary compounds consisting of two f-block metals, with the trend to D1 consisting of two s-block metals. The final cluster in D1 consists of the $\mathrm{XeCs}$ compounds from $\mathrm{XeCs}_{4}$ to $\mathrm{Xe}_{3} \mathrm{Cs}$. Following the trend to $\mathbf{B} 4$ we see additional compounds containing Y, Sc, and $\mathrm{Zr}$, with U/Pu-pnictogen binary compounds found in A3-A4.

Chemical Families:

- Left: Actinide + p-block/transition metal

- Central: Lanthanide/actinide f-f compounds these.

- Upper right: Lanthanide + lanthanide in the center to alkaline + alkaline to alkali + alkali, transitioning smoothly between

Composition Trends:

- Left: Generally $A_{0.8-0.95} B$ $A_{3} B$.

- Central: Large variance, greater actinide concentration to the left of $A_{9} B$, and higher lanthanide concentration in the center of

- Upper Right: Follows clusters of concentration through each of the chemical families, often to a high doping of one ion.

Chemical Trends:

- Left: Generally a high doping of $\mathrm{U} / \mathrm{Np} / \mathrm{Pu} / \mathrm{Th}$ and a transition metal

- Central: Stronger ratios of actinides on the far left, generally transitioning through actinide for all the f-f binary compounds

- Upper Right: Moving up this cluster each of the main clumps center around high concentrations of elements from each group laid out above. 


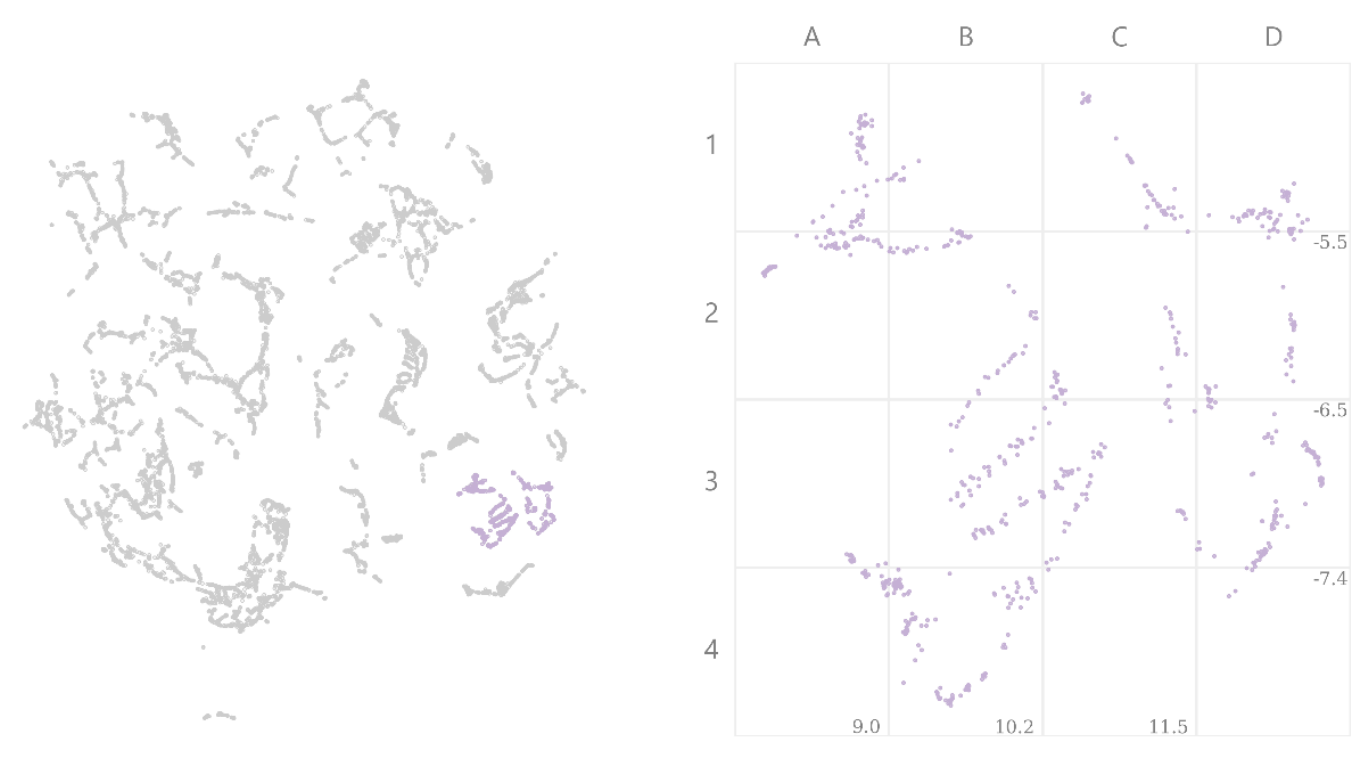

Label 11

Here we see a region of the map which due to chemical differences arguably should be separated into two clusters down the $\mathbf{C}$ column, but due to proximity have been classified under the same label with DBSCAN. The right hand side shows a natural continuation of cluster 9, following the same chemical families of s-block and f-block metals bonded to a p-block metal, with increasing $A$ ion ratio moving down the plot from $\mathrm{Th}_{2} \mathrm{Ge}(\mathbf{C 1})$ to $\mathrm{Ba}_{9} \mathrm{Ga}(\mathbf{D 4})$. From right to left the $B$ ion electronegativity decreases, which trend towards inter-metallic compounds, which are typical in the central and lower left regions of the global plot.

A1-B4 consists entirely of these inter-metallically bonded compounds, which are generally f-d valence bonded. From A1 we find these in the $A_{3} B_{2}$ ratio with $\mathrm{La}_{3} \mathrm{Ir}_{2}$, which increases in $A$ ion ratio as we move around the cluster clockwise until $\mathrm{La}_{3} \mathrm{Sn}$ at the bottom most point of $\mathbf{B 4}$, which come to more equal ratio to $A_{3} B$ in $\mathbf{A 3}$. For each of the roughly parallel lines, these show variation in elements, with an f-block $A$ ion and a d-block $B$ ion, with each of the distinct lines possessing a fixed ratio, which are $A_{2} B, A_{7} B_{3}$, and $A_{3} B$ respectively. Each of the $B$ ions throughout this cluster tend to be one of the following: $\mathrm{Ir}, \mathrm{Ru}, \mathrm{Ni}, \mathrm{Co}, \mathrm{Au}, \mathrm{Pt}, \mathrm{Pd}, \mathrm{Ni}, \mathrm{Tl}, \mathrm{Sn}$, $\mathrm{Al}$, and Ge.

Chemical Families:

- Upper Left: Lanthanide + boron/carbon group B ion

- Left: Lanthanide + alkali/transition metal from the right of group 8.

- Right: Lanthanide/alkaline/alkali + p-block element

Composition Trends:

- Upper Left: $A_{4} B_{3}$

- Left: Each approximately parallel lines consist of a single ratio of elements, moving down the cluster these are $A_{9} B_{11} \rightarrow A_{5} B_{4}$ $\rightarrow A_{3} B_{2} \rightarrow A_{2} B$ (first parallel line) $\rightarrow A_{7} B_{3}$ (second line) $\rightarrow A_{3} B$ (third line continuing to bottom of the cluster). The cluster to the bottom left trends from $A_{2} B$ to $A_{3}$.

- Right: $A_{2} B \rightarrow A_{9} B$ from top to bottom.

Chemical Trends:

- Upper Left: Lanthanide arsenides to the left, with $B$ ion increasing in electronegativity as we move down the cluster.

- Left: Lanthanide $A$ ion and a mixture of transition metals and metals ( $\mathrm{Ru} \rightarrow \mathrm{In}+\mathrm{Mn}$ ), $B$ ion trends to the right hand side of the table as we move down the cluster. Moves through 9/10/11 $B$ ion to s-block $A$ ion from upper left to bottom right.

- Right: The entirety of the top of this cluster is of the form $A_{2} B$, as with the left hand cluster the $A$ ion ratio increases as we move down this cluster, with the alkali/alkaline/lanthanide ratio increasing steadily to $A_{9} B$ with $\mathrm{Ba}_{0.909} \mathrm{Ga}_{0.091}$. 

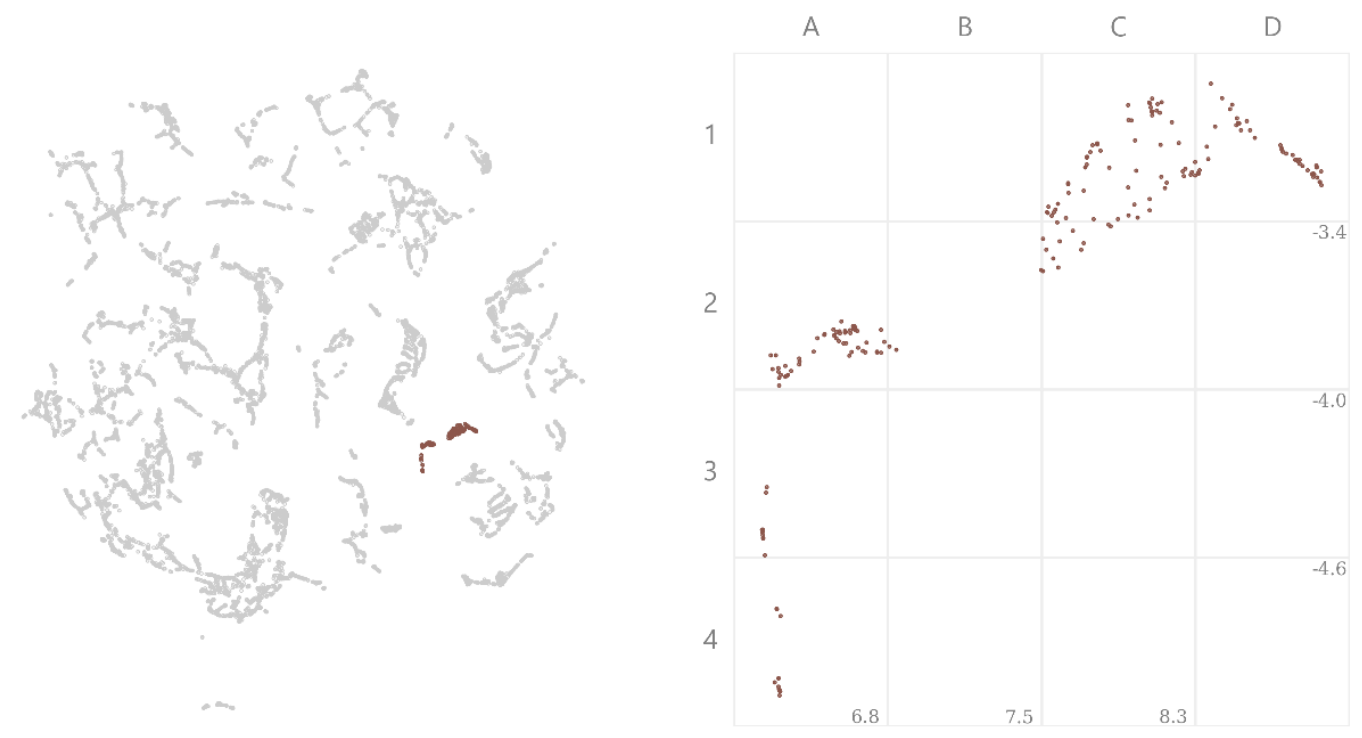

Label 12

This region continues the chemical trends of the previous cluster, being majority f-d bonded intermetallic compounds of the same elemental makeup. The trend in $B$ ion ratio is additionally preserved as we move further up, with almost all of these compounds being $A B$ binary compounds, barring the trailing points in the lower left, which transition smoothly to $A_{3} B$ as we rejoin cluster 11 .

Chemical Families:

- Left: Lanthanide/actinide + boron group/transition.

- Right: Lanthanide/alkaline/alkali + group 10/11.

Composition Trends:

- Left: $A_{3} B \rightarrow A B$

- Right: $A B$

Chemical Trends:

- Left: There is heavier $A$ doping towards the bottom of this cluster, with greater concentration of actinides. Moving up and to the right we see that the $B$ ions become more metallic.

- Right: Lanthanide/alkaline/alkali + p-block element. 

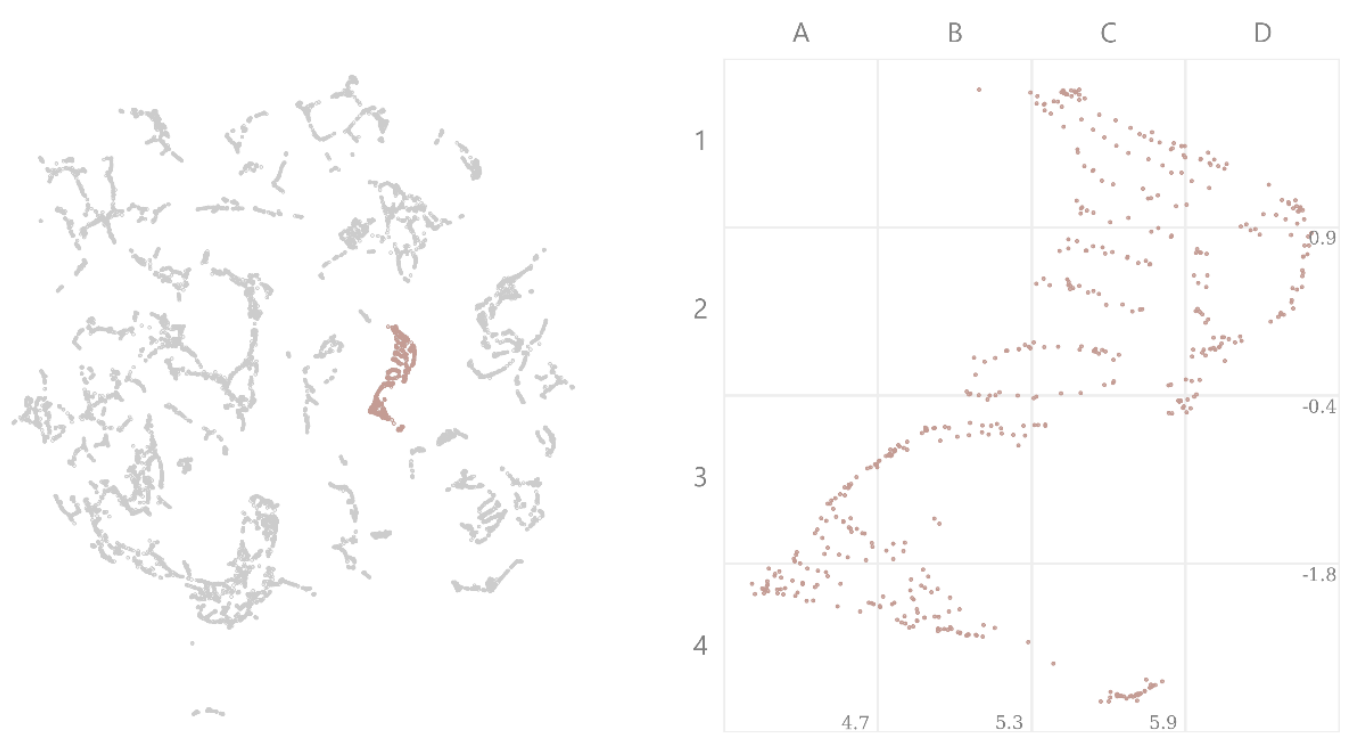

Label 13

Here we see the most prominent example of natural trends in substitutional feasibility being represented in graphical form. This cluster is almost exclusively $A B_{2}$ compounds, following a similar chemical trend to the previous two clusters. However with far greater numbers of reported stable compositions this allows for a more uniform arrangement of points which neatly follow trends in the Pettifor scale, see Figure 4 in the main body for a more thorough annotation.

Chemical Families:

- Lanthanide/actinide + transition metal.

Composition Trends:

- $A B_{2}$ with some trending to $A_{3} B_{4}$ in the bottom right.

Chemical Trends:

- This follows a very strong ratio clustering, with almost all compounds being of the form $A B_{2}$. The upper right of this cluster shows parallel lines, with each of these lines consisting of a lanthanides/actinide $A$ ion, and a single transition metal $B$ ion towards the upper right of the d-block. As we follow these lines to the east, these have the same $B$ ion, with the $A$ ion increasing in weight through the lanthanides and actinides. $B$ ion trends from zinc to the middle of transition metals from top to bottom. 

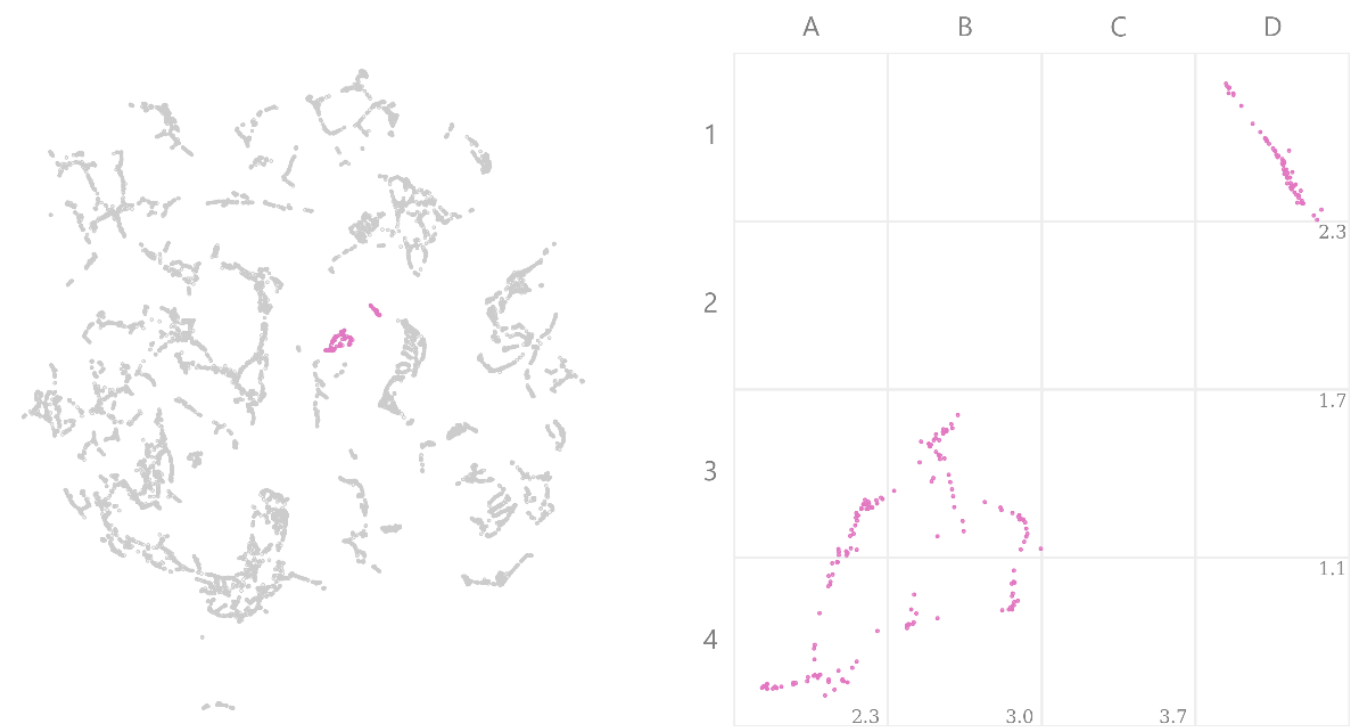

Label 14

The chemical trend of the previous group is again carried forward as a cluster of $A B_{3}$ ratio in D1. There are far fewer reported compositions with this ratio, and to the bottom left the trend continues to see a further increase in $B$ ion stoichiometry, possessing the same elements found in cluster 13 but with less chemical ordering, from $\mathrm{Y}_{0.207} \mathrm{Zn}_{0.793}$ at the upper tip of $\mathbf{B 3}$, to $\mathrm{Nd}_{0.143} \mathrm{Cd}_{0.857}$ at the bottom left of $\mathbf{A 4}$.

Chemical Families:

- Left: Lanthanide/alkaline/alkali $+\mathrm{Zn} / \mathrm{Cd} / \mathrm{Hg}$

- Right: Lanthanide/actinide $+\mathrm{Zn} / \mathrm{Cd} / \mathrm{Hg} / \mathrm{Mg}$

Composition Trends:

- Left: $A B_{6} \rightarrow A B_{4}$

- Right: $A B_{3}$

Chemical Trends:

- Left: $A$ ion is generally lanthanide, with some alkaline, alkali and actinide. Trends to increased $A$ ion ratio from left to right.

- Right: All of the form $A B_{3}$ following normal Pettifor trends with the noble gas inclusion of $\mathrm{XeMg}_{3}$. 

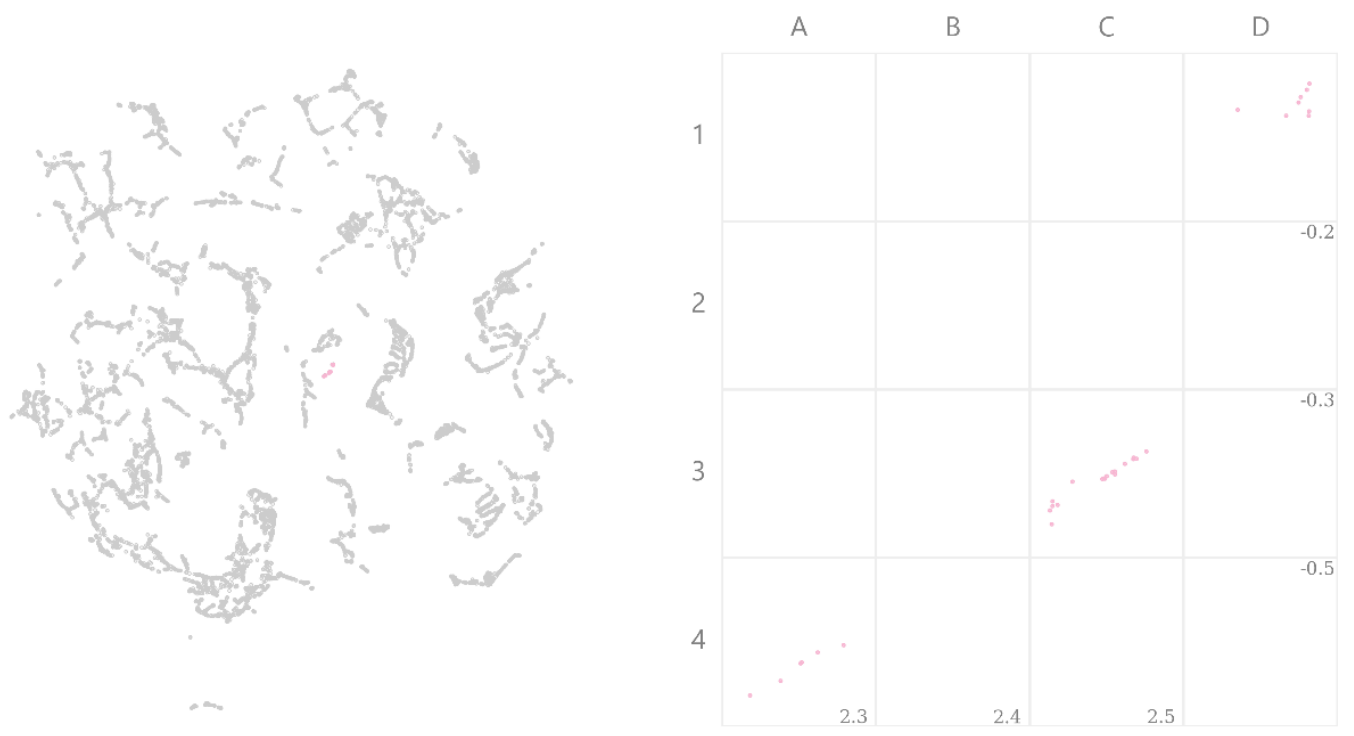

Label 15

This small cluster contains the entirety of the Mg metallically bonded compounds with a $B$ ion ratio greater than $79.3 \%$ going from $\mathrm{Ba}_{6} \mathrm{Mg}_{23}$ in $\mathbf{D 1}$, to $\mathrm{Y}_{16} \mathrm{Mg}_{109}$ in $\mathbf{A 4}$.

Chemical Families:

- Mg + s-block metal

Composition Trends:

• $A_{0.207} B_{0.793} \rightarrow A_{0.128} B_{0.872}$

Chemical Trends:

- Smooth variation of Mg doping from bottom to top of this cluster. 

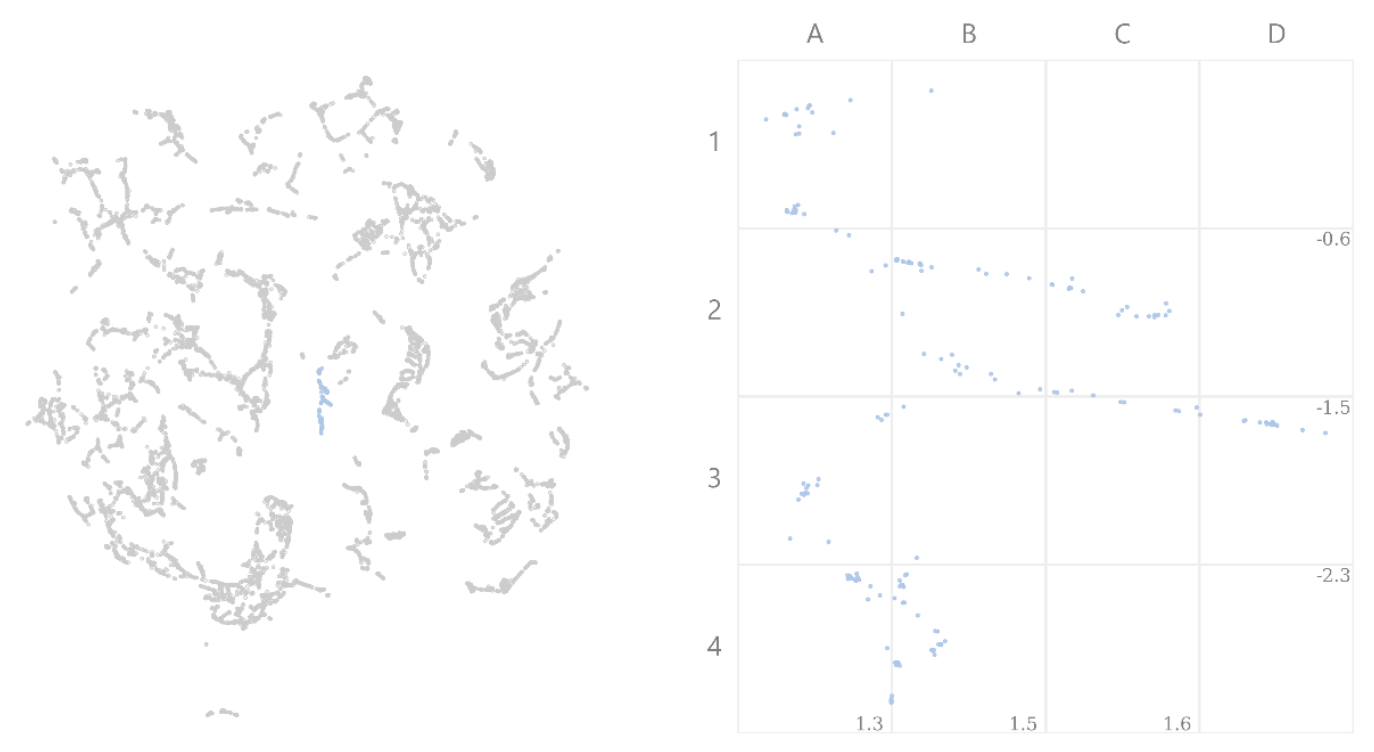

Label 16

We follow the continued decrease in $B$ ion ratio moving further down the cluster, from $A B_{7}$ in $\mathbf{A} \mathbf{1}$ reaching to $A_{0.077} B_{0.923}$ in $\mathbf{A 3}$, before following a small increase in $A$ ratio with $\mathrm{Fe} / \mathrm{Mn}$ bonded compounds to $\mathrm{PrFe}_{7}$ in $\mathbf{A 4}$. The two branches follow $\mathrm{Zn} / \mathrm{Mg}$ compounds with the ratios $A_{21} B_{179}$ and $A_{77} B_{923}$ respectively.

Chemical Families:

- f-block/s-block + Hg/Cd/Zn/Fe/Mn/Mg

Composition Trends:

- $A B_{7} \rightarrow A_{0.07} B_{0.93}$

Chemical Trends:

- $B$ ion goes to the left of the transition metals as we move down the cluster. $A$ ion follows alkaline/lanthanide/actinide 

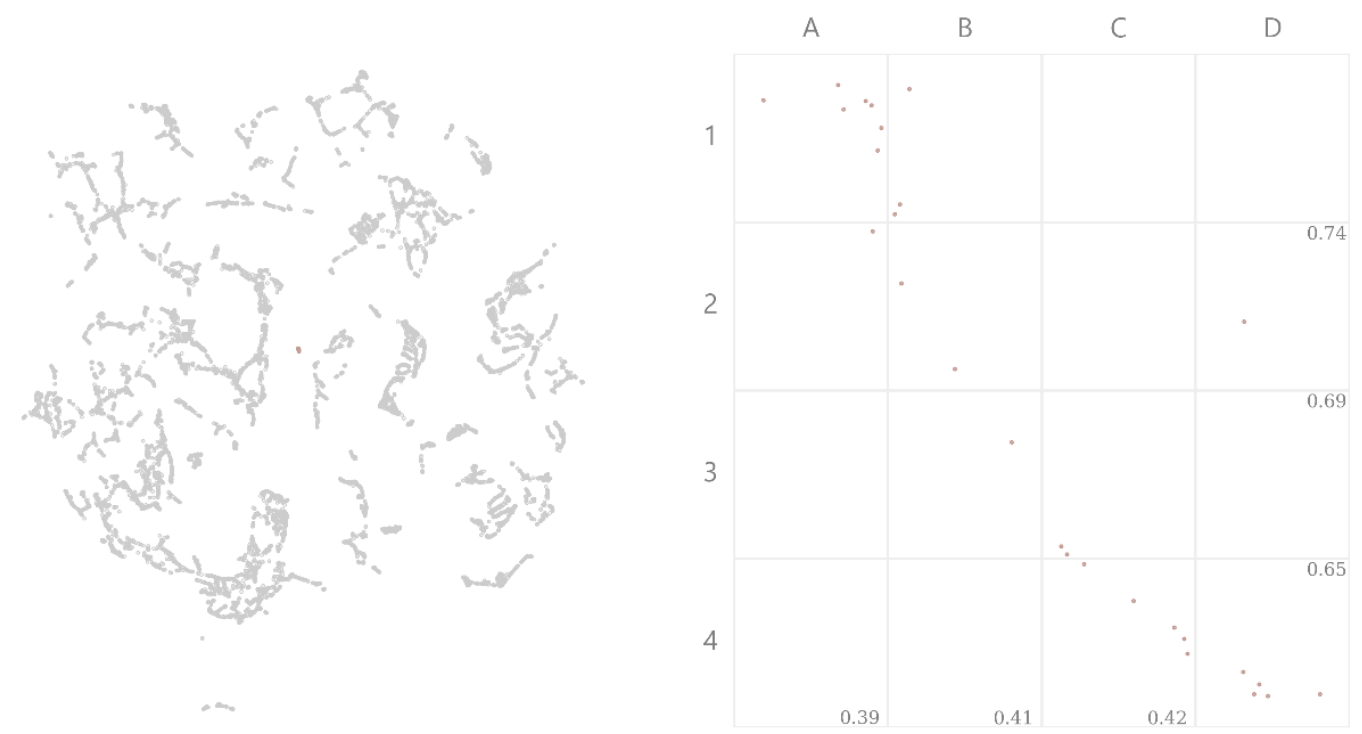

Label 17

This small cluster contains the entirety of the Be compositions in the ratio $A_{0.071} B_{0.929}$ where the $A$ ion is a lanthanide or actinide, which follows the same ratio as the compounds found at the closest point of cluster 16.

Chemical Families:

- Be + lanthanide/actinide

Composition Trends:

- $A_{0.071} B_{0.929}$

Chemical Trends:

- Cluster consisting of Be lightly doped with an f-block metal 

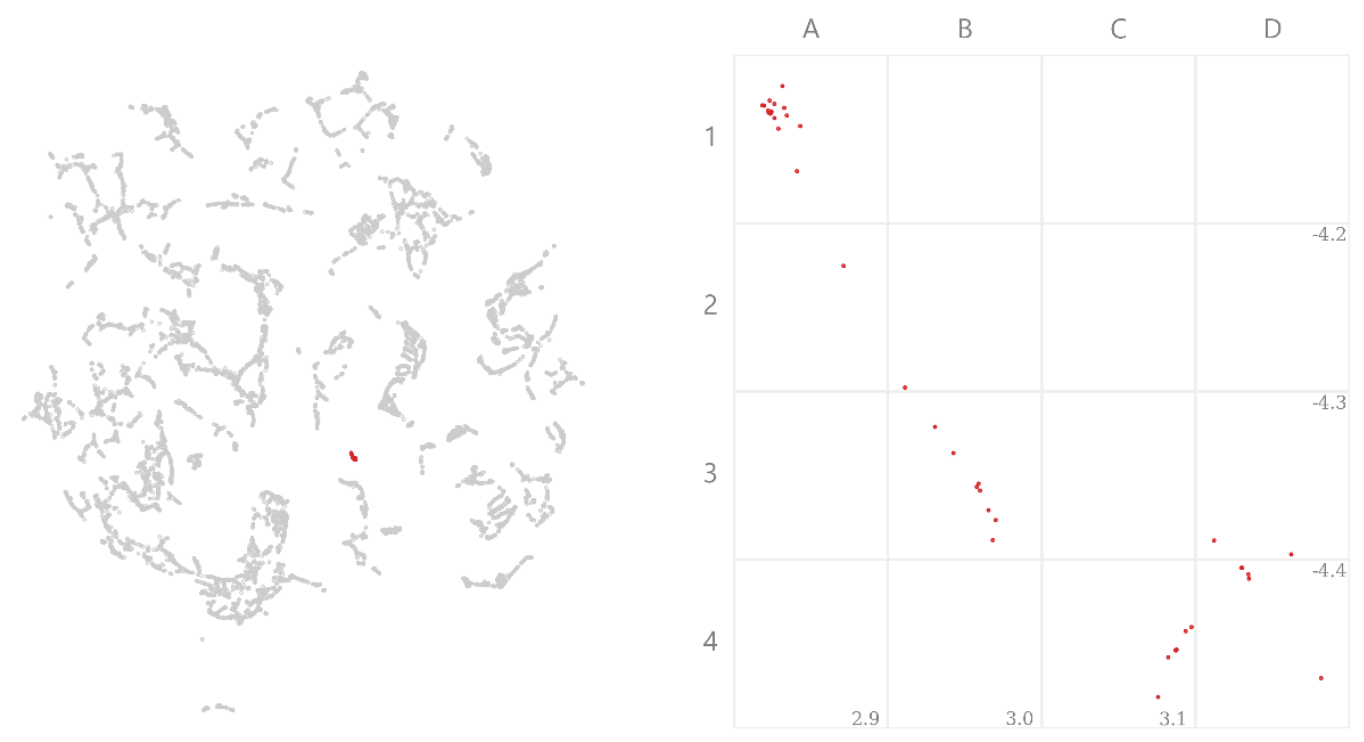

Label 18 family.

Here we have Mn (A1-A3) and $\mathrm{Fe}(\mathbf{C 4}-\mathbf{D} 4)$ binary compounds in the ratio $A_{0.207} B_{0.793}$ such that $A$ is a metal from the lanthanide

Chemical Families:

- Lanthanide + Fe/Mn

Composition Trends:

- $A_{0.207} B_{0.793}$ 

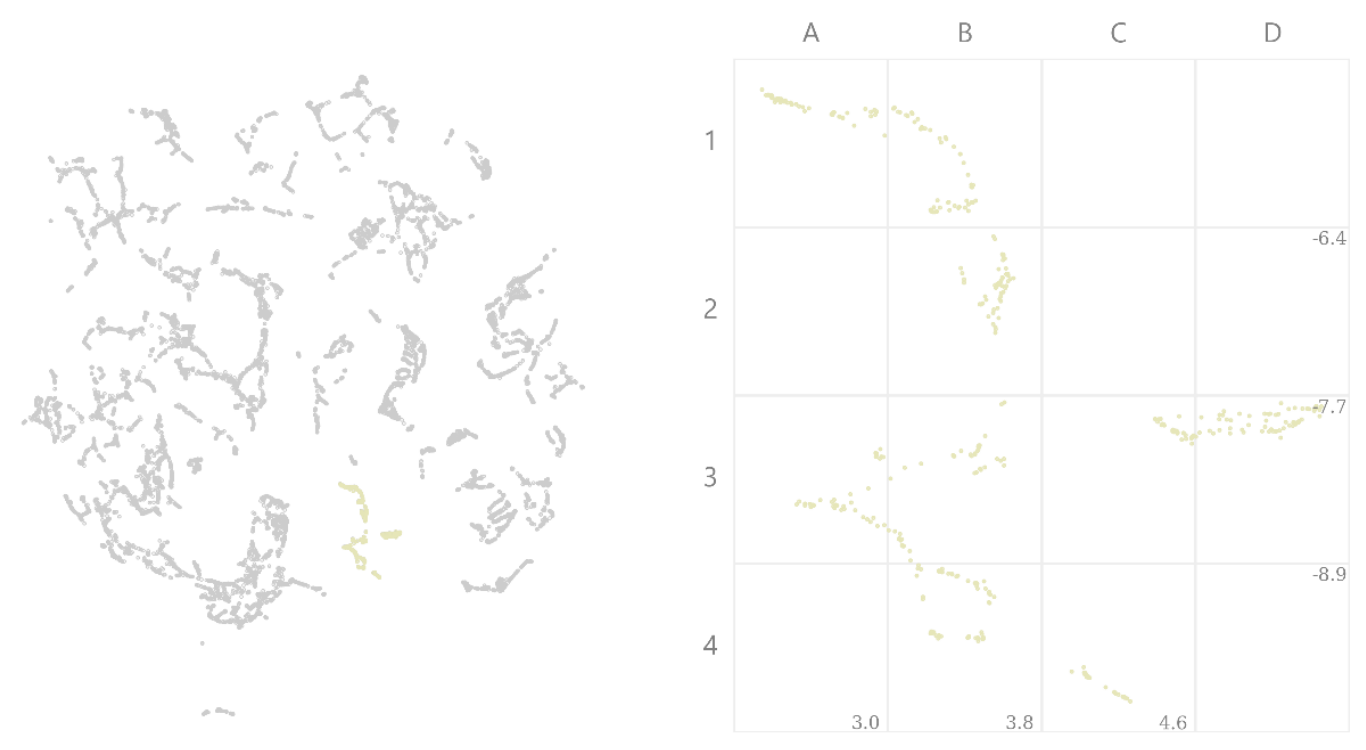

Label 19

Here we follow the trend of ratio for the lanthanide/actinides bonded with a transition metal, however we see a discrepancy in the ordering. The top of this cluster consists of $A B_{3}$ compounds trending to $A B_{5}$ in $\mathbf{C 4}$, which if the previous trends had been observed would place this between clusters 13 and 14. There is little similarity with cluster 20 to the left to justify this placement, which is a known flaw of the UMAP algorithm when plotting "islands" of datapoints, as these clusters do not have similar enough compositions with neighboring clusters to place these entirely correctly. The overall chemical similarities with the previous clusters is still observed however.

Chemical Families:

- Upper: Lanthanide/actinide $+\mathrm{Ni} / \mathrm{Co} / \mathrm{Cu}$

- Right: Lanthanide/actinide $+\mathrm{Rh} / \mathrm{Ir} / \mathrm{Pt} / \mathrm{Pd} / \mathrm{Au}$

- Lower: Lanthanide/actinide $+\mathrm{Pd} / \mathrm{Pt} / \mathrm{Cu} / \mathrm{Ag} / \mathrm{Au}$

Composition Trends:

- Upper: $A B_{3} \rightarrow A B_{4}$

- Right: $A B_{3}$

- Lower: $A B_{20} \rightarrow A B_{5}$

Chemical Trends:

- Upper: Going down the cluster, as $B$ increases in proportion, it trends to the right of the transition metals, with actinides mostly found towards the center.

- Right: Transitions through group 9/10/11 metals, from left to right in $B$ ion. $A$ ion moves through actinides then lanthanides.

- Lower: Towards the center and left we see very high $B$ ion concentration, towards the center of the d-block. $A$ ion is a disordered collection of lanthanides, actinides and alkaline metals. 


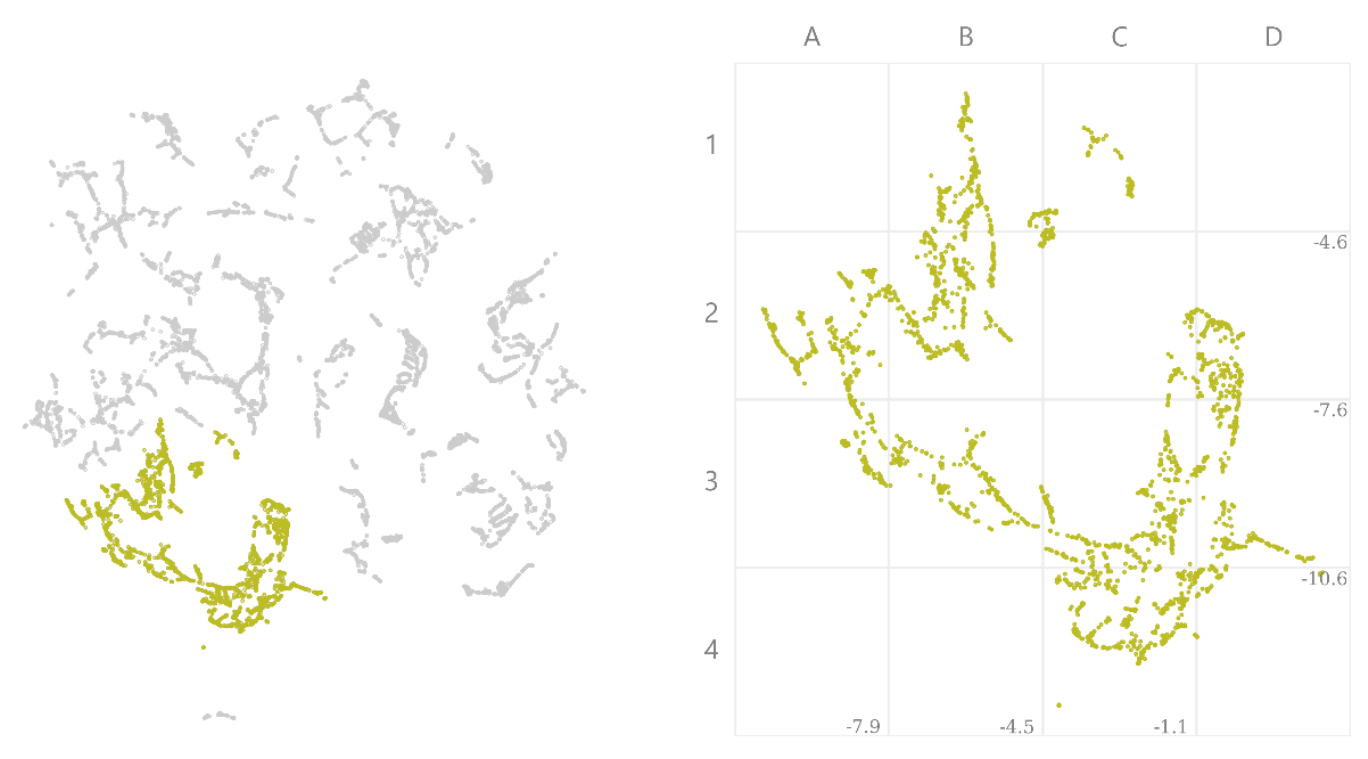

Label 20

The largest cluster, of 2729 compositions, is invariably dominated by the transition metal compounds, which are almost exclusively found in this region. Here we see a complete range of ratios, and the trends as we move around the crescent tend to follow swells in ratio for specific elements, from $A B$ compositions at $\mathbf{C 3}$ to DyCu $\mathbf{C u}_{99}$ at $\mathbf{A 2}$. There is a cluster of transition metals with more electronegative p-block elements at D2-D4 which generally shows a higher transition metal doping, from $A_{2} B$ to $A_{9} B$. The cluster from B1-B2 leading up is initially two transition metals in $A_{3} B$ ratio, before sharply increasing in transition metal doping as the $B$ ion moves through to the p-block metals. This ratio evens out moving further up, leading to the next group of $d-p$ bonded compounds.

Chemical Families:

- Right: Transition + transition

- Central Left: Transition + transition

- Upper Left: Transition + transition/p-block

- Upper Central: Transition $+\mathrm{Zn} / \mathrm{Cd} / \mathrm{Hg} / \mathrm{Be} / \mathrm{Ga} / \mathrm{Al}$

Composition Trends:

- Right: $A B \rightarrow A_{3} B$ from left to upper right. Lower cluster is mixture of $A B, A_{2} B, A_{2} B_{3}, A_{3} B$, and $A_{20} B$

- Central Left: From left, high $A$ ratio, $A_{9} B \rightarrow A B$ as we move down to the center.

- Upper Left: $A B \rightarrow A_{4} B \rightarrow A_{2} B$

- Upper Central: $A B \rightarrow A B_{99}$ and $A_{3} B_{5} \rightarrow A B$

Chemical Trends:

- Right: Transition + transition

- Central Left: High ratios of $\mathrm{Fe} / \mathrm{Ni} / \mathrm{Mn} / \mathrm{Co}$ at the left hand side of this region, transitioning smoothly in $A B$ distribution to the center of the cluster. This more sharply trends to an equal distribution as it follows the main cluster up.

- Upper Left: Moving up the cluster, the B ion migrates to the right of the periodic table through the p-block metals. The $B$ ion starts in small proportion, and increases as we move up the cluster.

- Upper Central: The left hand cluster are entirely Zn compounds increasing in concentration from left to right. The right hand cluster mostly contains binary compounds of transition metals from the very left of the d-block and p-block elements. 

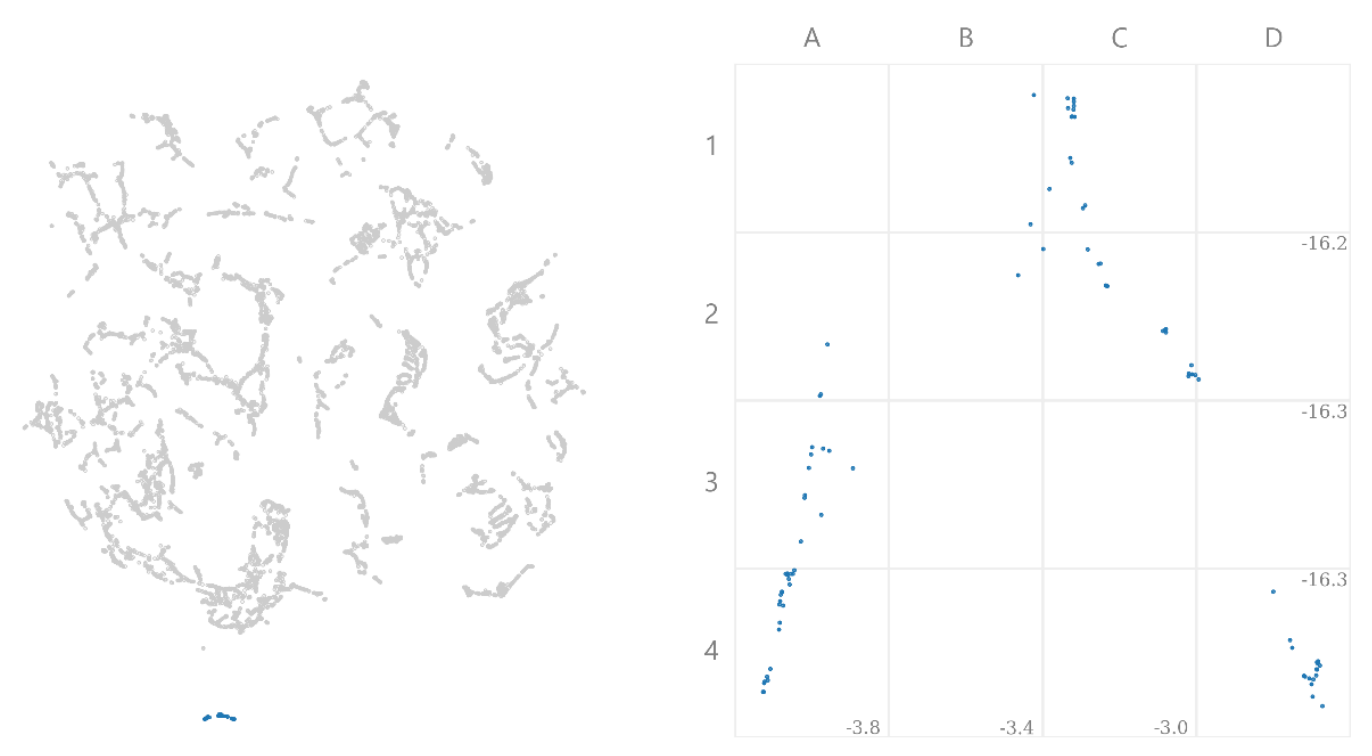

\section{Label 21}

These three isolated island each contain a distinct $A$ ion (being $\mathrm{Fe} / \mathrm{Co} / \mathrm{Ni}$ ) bonded to a lanthanide or actinide, in a reasonably wide range of ratios.

Chemical Families:

- $\mathrm{Fe} / \mathrm{Co} / \mathrm{Ni}+$ lanthanide/actinide

Composition Trends:

- $A B_{5} \rightarrow A_{0.071} B_{0.929}$, disordered

Chemical Trends:

- Three islands each of a high concentration of $\mathrm{Fe} / \mathrm{Co} / \mathrm{Ni}$, and separated by presence of this ion. $A$ ion either a lanthanide or actinide with no discernable trend. 

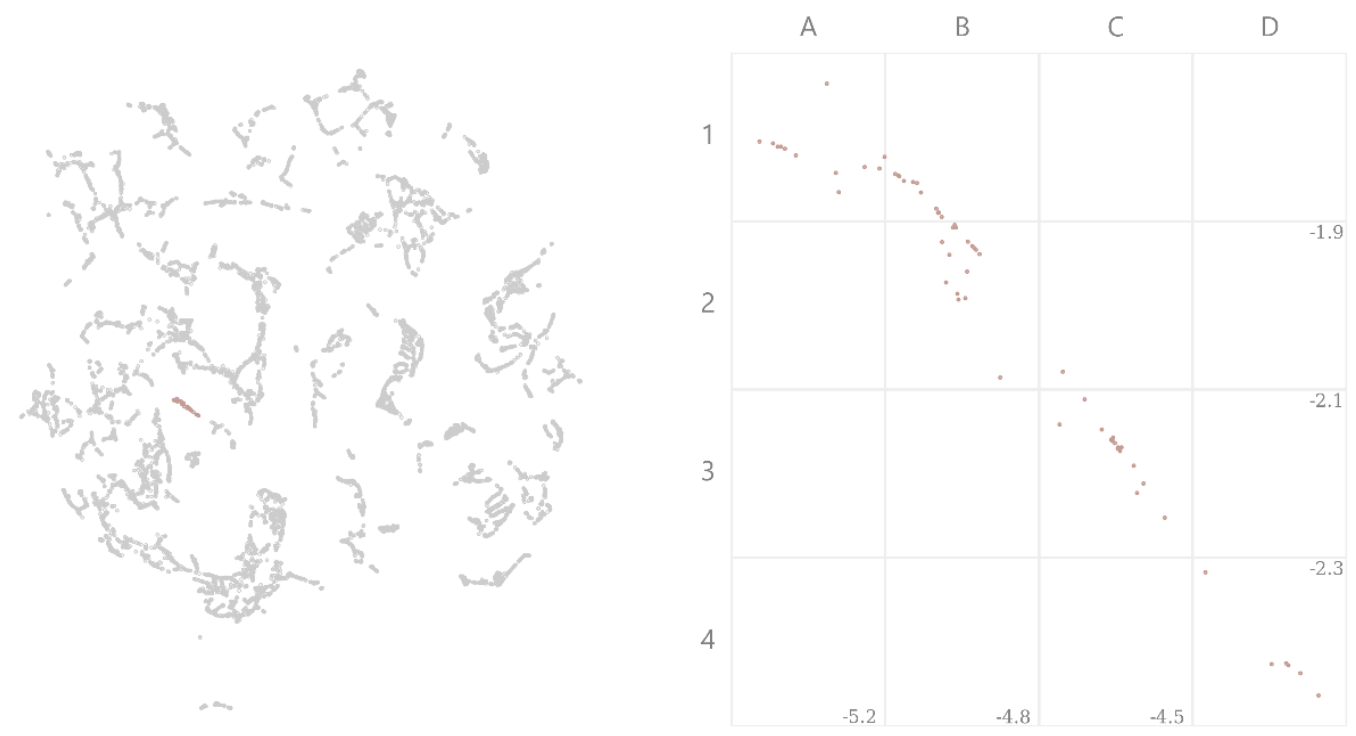

Label 22

Here we see a natural continuation of cluster 20 of d-p bonded compounds which have initially higher transition metal doping of $A B_{2}$ closer to the transition metal cluster, which leads to $A B$ compounds as we go further towards $\mathbf{D 4}$.

Chemical Families:

- Transition metal + $\mathrm{Be} / \mathrm{Al} / \mathrm{Ge}$

Composition Trends:

- $A B_{2} \rightarrow A B$, upper left to bottom right

Chemical Trends:

- As we move down the trend we see the left hand side transition metals plus $\mathrm{Be} / \mathrm{Al} / \mathrm{Ga}$ (in that order) 

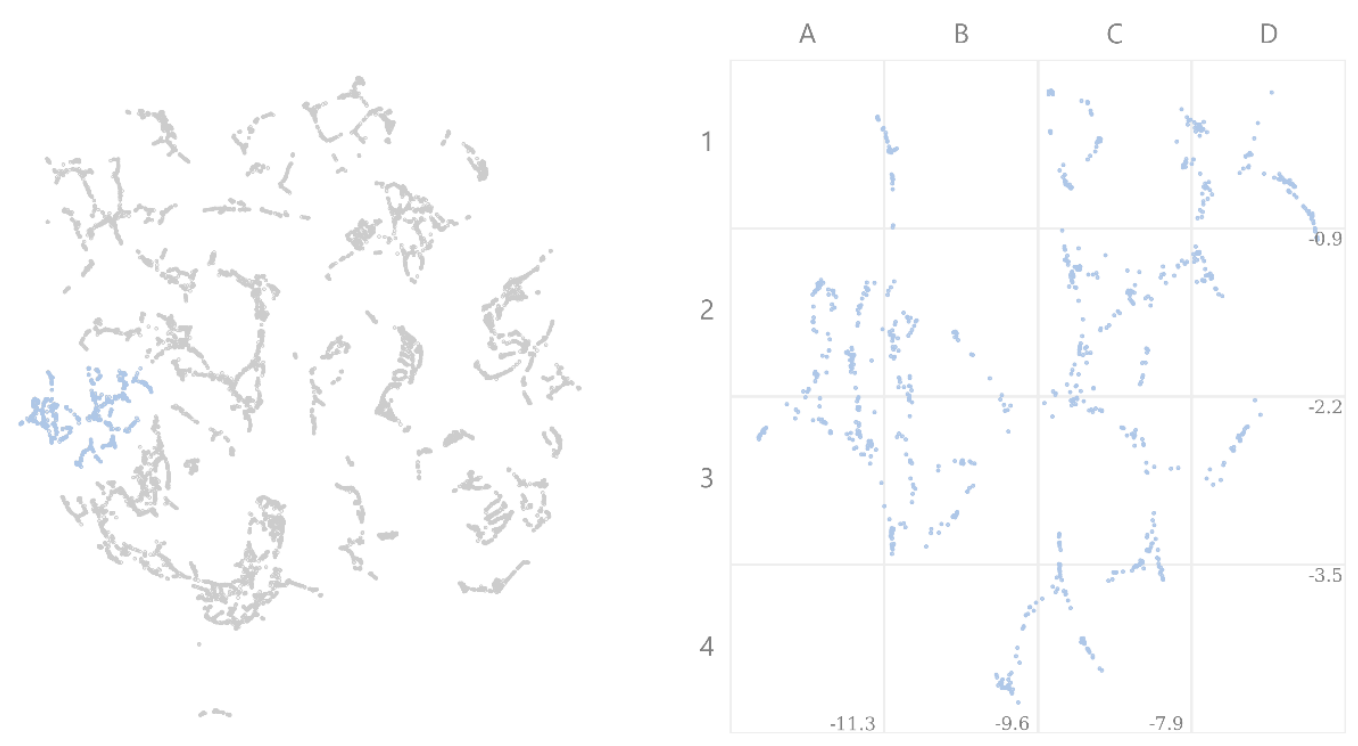

Label 23

We again continue the chemical trends of cluster 20 of high doping transition metal $A$ ion with a p-block metal across B4-D3. As expected the ratio of $B$ ions increases as we move towards cluster 0 , with this being a post-transition metal. Each of the branches tend to contain distinct $B$ ions $(\mathrm{Ga} / \mathrm{Ba} / \mathrm{Be} / \mathrm{Al} / \mathrm{In} / \mathrm{Tl})$ and follow local trends in ratio, with $\mathrm{AuTl}_{3}$ found in D1.

Chemical Families:

- Left: Transition metal + p-block

- Lower: Transition metal $+\mathrm{Hg} / \mathrm{Cd} / \mathrm{Mg} / \mathrm{Cu}$

- Upper Right: Transition metal $+\mathrm{Mg} /$ boron group

Composition Trends:

- Left: $A B \rightarrow A_{0.92} B_{0.08}$ increasing smoothly down lines, $A B$ to the right

- Lower: $A_{19} B \rightarrow A B$ as we move from bottom to the center

- Upper Right: $A B$ in the center. Trends to $A B_{19}$ directly up, and to $A B_{3}$ diagonally to the right

Chemical Trends:

- Left: High concentration of central d-block, mostly Fe and Co in the center, with branching into Ni/Co, Fe, Mn, Mg. The $A$ ion trends follow the four roughly parallel lines down the cluster. The $B$ ions tend to be from the boron, carbon and nitrogen groups, slightly increasing in atomic weight as we move to the right of the cluster. The top of the cluster is characterized by a halogen $B$ ion with a transition metal in groups 9/10/11.

- Lower: Follows cluster to the left from $A B$ increasing to a majority $A$ ion ratio as we follow the cluster down. $A$ is $\mathrm{Cd}, \mathrm{Mg}$, and $\mathrm{Hg}$ with a right hand side transition metal. At the very bottom this cluster the $B$ ion moves further to the right of the table to give a selection of high $\mathrm{Mg}$ compounds.

- Upper Right: This follows the trend of increasing $B$ ion ratio, with the $B$ ion trending from magnesium to aluminum/beryllium. The transition metal trends further to the left of the periodic table as we move up the cluster. A high concentration of metal towards the upper perimeters of the cluster, including Be/Al majority compounds. 


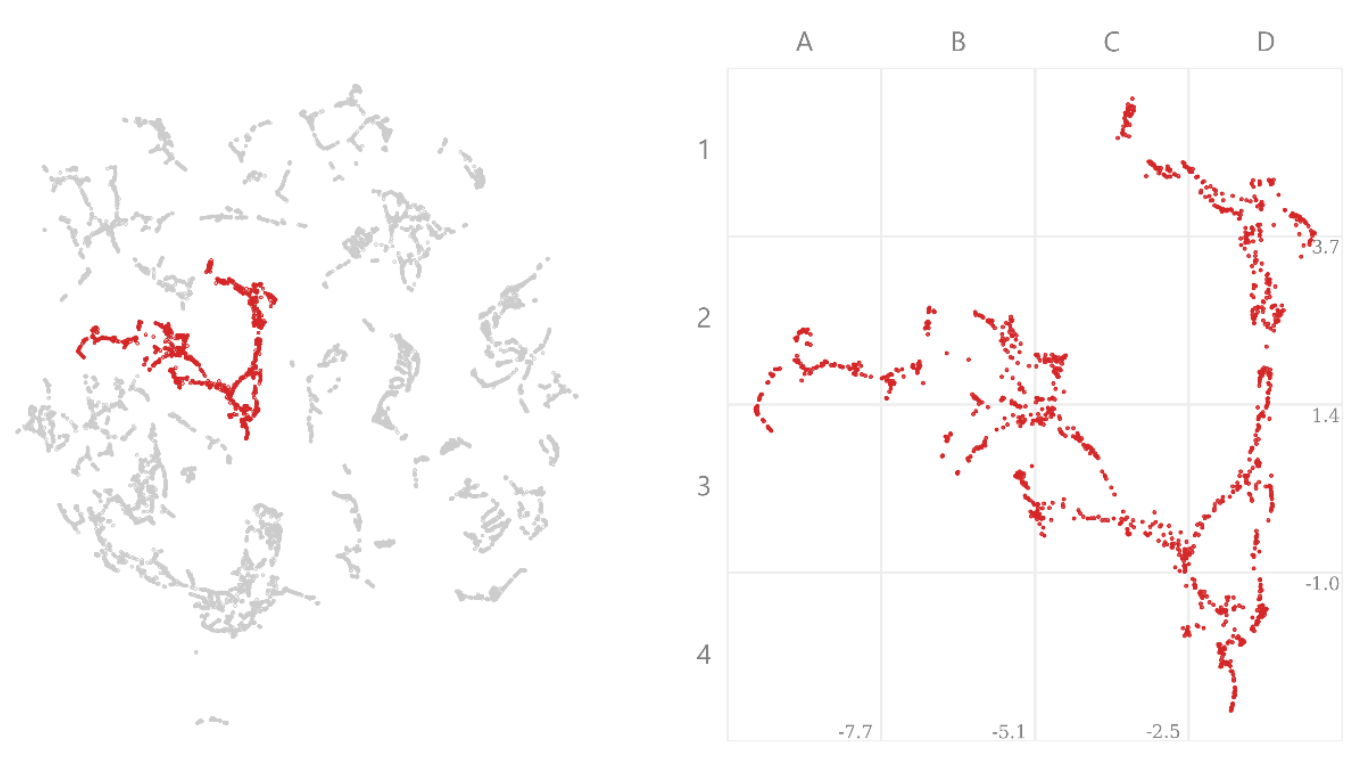

Label 24

Here we find the second largest cluster of compositions, which is almost entirely transition metals bonded with p-block metalloids and non-metals. From A2 to D4 we find mostly $A B$ compositions which trend to higher $B$ concentration as we move towards $\mathbf{C 2}$. The $B$ ion electronegativity increases as we follow the crescent up, through the pnictogens and chalcogens, with $\mathrm{CoO}_{2}$ found in $\mathbf{C 1}$. Here we see that the branch at D1 follows some of the halogens, suggesting that cluster 2 may have been misplaced by UMAP, as a more natural placement would follow to the right of this, before following on to cluster 3.

Chemical Families:

- Left: Transition metal/p-block + p-block

- Lower Right: Transition metal + p-block

- Upper Right: Transition metal + p-block

Composition Trends:

- Left: $A B_{19} \rightarrow A_{3} B_{2} \rightarrow A B \rightarrow A B_{2} \rightarrow A_{2} B_{3} \rightarrow A B$

- Lower Right: $A B \rightarrow A_{3} B_{2} \rightarrow A_{5} B_{3}$, going down

- Upper Right: $A B \rightarrow A_{2} B_{3} \rightarrow A B_{2}$

Chemical Trends:

- Left: This cluster begins with almost pure aluminum, with the dopants quickly becoming a d-block element in more equal ratios. This is initially a period 4 transition metal + p-block, but has group 9/10/11 metals + p-block as we move further to the right, or $\mathrm{Mg} / \mathrm{Be}+\mathrm{p}$-block as we move further up. Towards the center are 9/10/11 metals + a lower left p-block. The $A$ ions move to the left of the d-block as we keep moving to the right, and the $B$ ion moves to the top of the p-block of the general form $A B_{2}$. As we progress to the lower right these return to $A B$ nature with a left hand d-block and $\mathrm{B} / \mathrm{C} / \mathrm{P} / \mathrm{Si} / \mathrm{As} / \mathrm{Sb}$.

- Lower Right: As we keep moving down the cluster this increases in $A$ metal content to $A_{5} B_{3}$, of a left hand d-block + a carbon/pnictogen group. As we follow this up and to the right, the $A B$ ratio remains more consistent, and the $B$ ion moves towards the chalcogens (with a left hand d-block). Chalcogen content increases steadily as we move up the cluster to around $A_{11} B_{14}$ at the thinnest point as we move up

- Upper Right: Moving up, the $B$ ion concentration increases to around $A B_{2}$. This consists of mostly transition metals (plus some alkaline and lanthanide) $A$ ion and chalcogen. Further up the cluster, the $A$ ion trends to the right of the d-block. As we move to the far right of the cluster, we get the left hand d-block metals and a halogen equal ratio. 

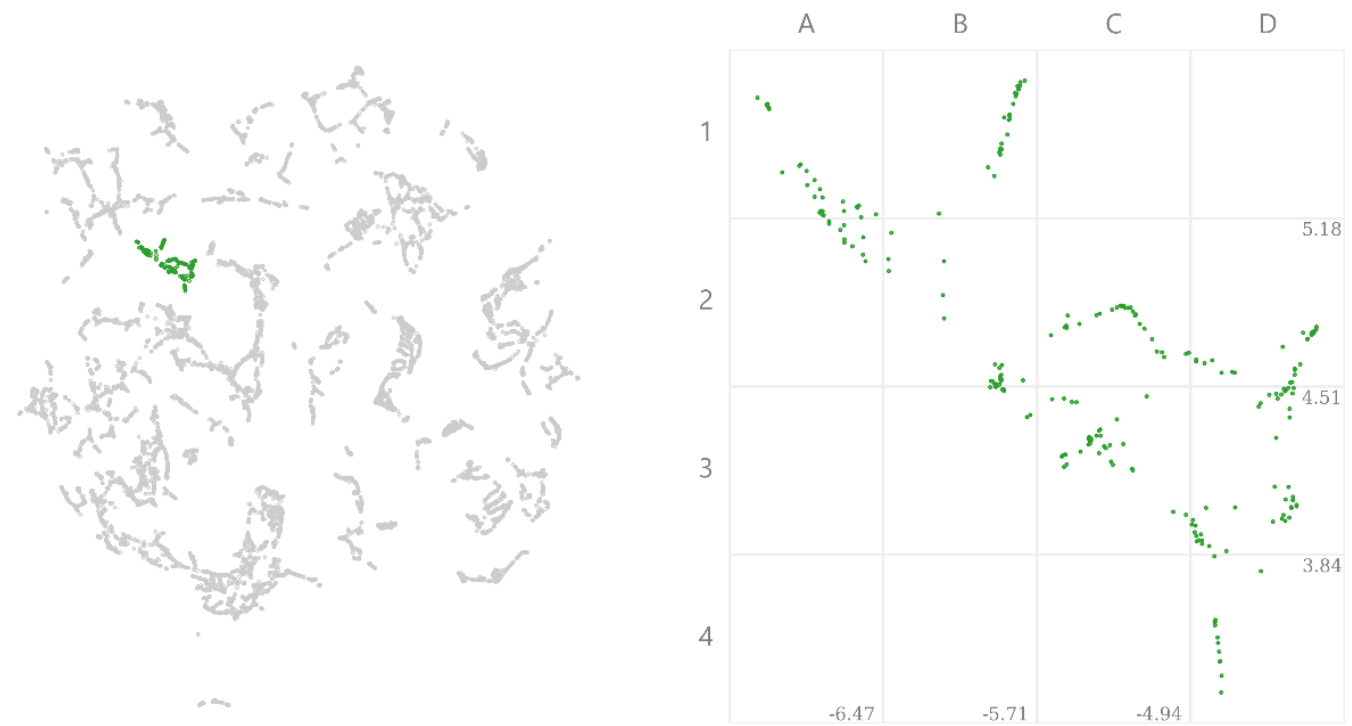

Label 25

The final cluster initially follows the trend from cluster 24, with transition metal $A B$ chalcogenides found from $\mathbf{C 3}$ to $\mathbf{D} 4$. The transition metal shows an increase in electronegativity as we move towards cluster 0 as we would expect. With $\mathrm{AlO}$ at $\mathbf{A 1}$ we see the beginning of the p-p valence bonded binary compounds which makeup the entirety of the upper left region in cluster 0 .

Chemical Families:

- Transition metal/actinide + chalcogen/halogen

Composition Trends:

- $A B \rightarrow A_{2} B_{3}$ in the far right

Chemical Trends:

- From the top left we have transition metals from the right of d-block $A$ ions, in equal ratio to a halogen. As we move down and to the right, the $A$ ion moves further of the left of the d-block to the lanthanides, with the $B$ ion being a chalcogen element. Further to the bottom right we see a small increase in $B$ ion ratio, which is mostly a chalcogen or pnictogen (often $\mathrm{O}$ ) 


\section{References}

[1] Alexandrov, A. D. A theorem on triangles in a metric space and some of its applications. Trudy Mat. Inst. Steklov. 1951, 38, pp 5-23.

[2] Ambrosio, L.; Gigli, N.; Savaré, G. Gradient flows: in metric spaces and in the space of probability measures. 2008, Springer Science \& Business Media.

[3] Villani, C. Optimal Transport. 2008, Springer Science \& Business Media.

[4] Pettifor, D. G. The structures of binary compounds. I. Phenomenological structure maps. J. Phys. C Solid State Phys, 1986, 19, pp 285-313. doi.org/10.1088/0022-3719/19/3/002

[5] Villars, P.; Brandenburg, K.; Berndt, M.; LeClair, S.; Jackson, A. ; Pao, Y.-H.; Igelnik, B.; Oxley, M.; Bakshi, B.; Chen, P.; Iwata, S. Binary, ternary and quaternary compound former/nonformer prediction via Mendeleev number. J. Alloys Compd, 2001, 317-318, pp 26-38, doi.org/10.1016/S0925-8388(00)01410-9

[6] Glawe, H.; Sanna, A.; Gross, E. K. U.; Marques, M. A. L. The optimal one dimensional periodic table: a modified Pettifor chemical scale from data mining. New J. Phys. 2016, 18(9), 093011, doi.org/10.1088/1367-2630/18/9/093011.

[7] Ward, L.; Agrawal, A.; Choudhary, A.; Wolverton, C. A general-purpose machine learning framework for predicting properties of inorganic materials. 2016, Npj Comput. Mater, 2, 16028, doi.org/10.1038/npjcompumats.2016.28 